\title{
A genome-wide almanac of co-essential modules assigns function to uncharacterized genes
}

Michael Wainberg ${ }^{1,2,4}$, Roarke A. Kamber ${ }^{1,4}$, Akshay Balsubramani ${ }^{1,4}$, Robin M. Meyers ${ }^{1}$, Nasa Sinnott-Armstrong ${ }^{1}$, Daniel Hornburg ${ }^{1}$, Lihua Jiang ${ }^{1}$, Joanne Chan ${ }^{1}$, Ruiqi Jian ${ }^{1}$, Mingxin $\mathrm{Gu}^{1}$, Anna Shcherbina ${ }^{1}$, Michael M. Dubreuil ${ }^{1}$, Kaitlyn Spees ${ }^{1}$, Michael P.

Snyder ${ }^{1}$, Anshul Kundaje ${ }^{1,2 *}$, Michael C. Bassik ${ }^{1,3, *}$

1. Department of Genetics, Stanford University, Stanford, California 94305-5120, USA

2. Department of Computer Science, Stanford University, Stanford, CA, 94305

3. Chemistry, Engineering, and Medicine for Human Health (ChEM-H), Stanford University, Stanford, California 94305-5120, USA.

4. These authors contributed equally

*Correspondence to akundaje@stanford.edu or bassik@stanford.edu 


\section{SUMMARY}

1 A central remaining question in the post-genomic era is how genes interact to form biological

2 pathways. Measurements of gene dependency across hundreds of cell lines have been used to

3 cluster genes into 'co-essential' pathways, but this approach has been limited by ubiquitous false

4 positives. Here, we develop a statistical method that enables robust identification of gene co-

5 essentiality and yields a genome-wide set of functional modules. This almanac recapitulates

6 diverse pathways and protein complexes and predicts the functions of 102 uncharacterized

7 genes. Validating top predictions, we show that TMEM189 encodes plasmanylethanolamine

8 desaturase, the long-sought key enzyme for plasmalogen synthesis. We also show that C15orf57

9 binds the AP2 complex, localizes to clathrin-coated pits, and enables efficient transferrin uptake.

10 Finally, we provide an interactive web tool for the community to explore the results

11 (coessentiality.net). Our results establish co-essentiality profiling as a powerful resource for

12 biological pathway identification and discovery of novel gene functions. 


\section{INTRODUCTION}

14 A fundamental and still largely unresolved question in biology is how finite numbers of genes

15 generate the vast phenotypic complexity of cells and organisms (Barabási and Oltvai, 2004;

16 Chuang et al., 2010). With the understanding that modules of interacting genes represent a key

17 layer of biological organization, the complete identification of such functional modules and their

18 constituent genes has emerged as a central goal of systems biology (Costanzo et al., 2016;

19 Hartwell et al., 1999; Horlbeck et al., 2018; Stuart et al., 2003). However, efforts to map genetic

20 interactions and biological modules at genome scale have been hindered by the enormous

21 number of possible gene-gene interactions: assaying all pairs of genetic interactions among the

22 approximately 20,000 human genes (Harrow et al., 2012) would require 200 million distinct

23 readouts. Furthermore, despite substantial progress in elucidating the functions of individual

24 genes in recent decades through both targeted studies and unbiased approaches (Alonso and

25 Ecker, 2006; Carpenter and Sabatini, 2004; Mohr et al., 2014; Shalem et al., 2015), hundreds of

26 human genes remain functionally uncharacterized.

28 Pioneering work in yeast measured pairwise genetic interactions in high throughput by quantifying 29 the fitness of double knockout strains (Tong, 2004; Tong et al., 2001); more recently, this work 30 has been extended into a genome-wide map of yeast genetic interactions and modules (Costanzo 31 et al., 2010, 2016). In human cells, which unlike yeast cannot be crossed to generate double32 knockout mutants, a key advance towards genetic interaction mapping has been the development 33 of genome-scale CRISPR/Cas9 and RNAi screens (Mohr et al., 2014; Shalem et al., 2015) which 34 have been repurposed to perform pairs of perturbations (Bassik et al., 2013; Boettcher et al., 35 2018; Du et al., 2017; Han et al., 2017; Horlbeck et al., 2018; Rosenbluh et al., 2016; Shen et al., 36 2017). Yet despite considerable successes, double-perturbation genetic interaction mapping is 37 inherently limited by the combinatorial explosion of gene pairs, with the largest human genetic 38 interaction map to date (Horlbeck et al., 2018) having only assayed 222,784 gene pairs, or $\sim 0.1 \%$ 
of all possible genetic interactions, thus far precluding the generation of a genome-wide map of

40 functional modules in human cells.

42 A complementary approach that circumvents this limitation is to measure the fitness of single-

43 gene perturbations across multiple conditions, and map putative functional interactions by

44 correlating the resulting phenotypic profiles (Figure S1A), referred to as co-essentiality mapping.

45 Both co-essentiality mapping and genetic interaction mapping measure gene essentiality across

46 many different genetic backgrounds, but whereas the background for genetic interaction mapping

47 is the knockout of a single partner gene, for co-essentiality mapping it is the mutational and

48 transcriptional profile of a cell line. Co-essentiality mapping across diverse cancer cell lines has

49 recently been used to group genes into pathways and in some cases has identified novel gene

50 functions (Boyle et al., 2018; Kim et al., 2019; McDonald et al., 2017; Pan et al., 2018; Rauscher

51 et al., 2018; Wang et al., 2017).

53 Co-essentiality mapping, however, has its own fundamental limitation: unlike double-perturbation

54 mapping, where each pair of gene knockouts is independent, measurements in two different cell

55 lines may be strongly related, for instance because some pairs of cell lines are derived from the

56 same tissue or lineage. Existing approaches fail to account for violations of independence, leading

57 to inflated $p$-values, incorrect determinations of statistical significance, and an inability to identify

58 gene co-essentiality relationships in a robust, systematic manner (Figure S1B). In this study, we

59 address this critical limitation of co-essentiality mapping with a novel statistical method that

60 explicitly accounts for the non-independence of cell lines. We apply the method to a dataset of

61 genome-wide CRISPR screens in 485 diverse cancer cell lines (Tsherniak et al., 2017) and find

62 significantly improved enrichment for known pathway interactions and protein complexes.

63 
64 We find that these analytical advances greatly improve our ability to detect bona fide functional

65 modules. We generate a genome-wide almanac of co-essential modules, which both recapitulate

66 diverse known pathways and protein complexes and nominate putative functions for 102 poorly

67 characterized genes. We experimentally validate two such genes: we identify TMEM189 as the

68 gene encoding the plasmanylethanolamine desaturase (PEDS) orphan enzyme required for

69 synthesis of plasmalogen lipids, one of the most abundant lipid classes in the human body; and

70 we discover a role for $\mathrm{C} 15$ orf57 in regulating clathrin-mediated endocytosis. Finally, to accelerate

71 further biological discovery using this resource, we present an interactive web tool that enables

72 visualization and analysis of co-essential gene pairs and modules. 


\section{RESULTS}

\section{A genome-wide map of co-essential interactions}

75 To map co-essential interactions across compendia of genome-wide screens while accounting

76 for non-independence of cell lines, we devised a novel approach based on generalized least 77 squares (GLS), a classic statistical technique (Aitkin, 1935) (see Methods). We applied the

78 approach (Figure 1A) to a dataset of CRISPR screens in 485 cell lines from the Achilles project

79 (Tsherniak et al., 2017), with gene-level essentiality scores corrected for copy number and guide

80 efficacy using the CERES algorithm (Meyers et al., 2017). We noted the remarkably effective

81 statistical calibration of the method. Since the percentage of gene pairs expected to have

82 detectable functional interactions is much less than $50 \%$ (Horlbeck et al., 2018), the median $p$ -

83 value across gene pairs ought to be very close to 0.5 for a well-calibrated method. Indeed, we

84 found that the median GLS $p$-value was 0.48 , indicating near-perfect calibration, while the median

85 Pearson correlation $p$-value on the same dataset was 0.21 , indicating substantial inflation and

86 false-positive co-essential gene pairs (Figure 1B). We provide each gene's significant co-

87 essential interactors at a false discovery rate of $10 \%$ (Table S1).

89 Even while correcting for $p$-value inflation, GLS still has substantial power to detect co-essential

90 interactions. Around $80 \%$ of genes have at least one co-essential partner at $10 \%$ FDR (Figure

91 S2), and $40 \%$ of genes have at least ten partners: in all, we detect 93,575 significant co-essential

92 gene pairs. $99.4 \%$ of all partners are positively correlated, with the remaining $0.6 \%$ negatively

93 correlated. We noted that in many cases, negative correlations occur when one gene negatively

94 regulates the other: for instance, TP53 is negatively correlated with $M D M 2\left(p=1 \times 10^{-12}\right)$, which

95 ubiquitinates p53 to mark it for degradation (Moll and Petrenko, 2003); HER2 is negatively 96 correlated with PHLDA2 $\left(p=5 \times 10^{-6}\right)$, which was recently shown to inhibit HER2 signaling (Wang

97 et al., 2018); and MAPK1 is negatively correlated with DUSP6 $\left(p=2 \times 10^{-6}\right)$, a phosphatase that

98 inactivates several MAP kinases including MAPK1 (Furukawa et al., 2008). A second class of 
negative correlation arises from genes with similar functions that are active in mutually exclusive cell types, such as MYC and MYCN $\left(p=3 \times 10^{-11}\right)$ (Rickman et al., 2018).

Crucially, even though more essential genes tend to have more partners, $70 \%$ of the $10 \%$ least essential genes have at least one partner at 10\% FDR, and nearly half of these least essential genes have at least one partner at 1\% FDR (Figure S2). This suggests that, rather than being limited to detecting interactions among only strongly essential genes, the focus of previous coessentiality mapping efforts (Kim et al., 2019), co-essentiality is a genome-wide tool for pathway mapping.

We developed a method to visualize genes in a genome-wide interaction map based on their coessentiality profiles by placing more strongly co-essential gene pairs closer together, inspired by similar visualizations based on yeast genetic interaction maps (Costanzo et al., 2010, 2016). We found that naive application of dimensionality reduction techniques such as Principal Component Analysis (PCA) and Uniform Manifold Approximation and Projection (UMAP) (Mclnnes et al.,

114 2018) failed to effectively expose functional relationships between genes due to difficulty modeling the multi-scale nature of the co-essentiality network; previous attempts at visualizing coessentiality networks (e.g. McDonald et al., 2017) also suffer from a similar lack of discernible

117 structure. Instead, we first applied diffusion maps (Coifman and Lafon, 2006), a technique from 118 spectral graph theory, to separate coarse- and fine-scale components before applying UMAP (see 119 Methods). To further improve the layout, we incorporated module membership (defined below) 120 into the diffusion map in addition to pairwise co-essentiality. To showcase the power of this 121 approach, we manually annotated 39 'neighborhoods' within the interaction map highly enriched 122 for a particular pathway or complex (Figure 1C, D); collectively, these pathways and complexes 123 encompass many of the major aspects of cell biology. 
We next investigated whether the improved calibration of GLS translated into improved

127 prioritization of co-functional gene pairs. To do this, we used an established benchmarking 128 strategy (Pan et al., 2018) to measure how accurately GLS could recall the top 1 to 10 interaction 129 partners of each gene when compared with Pearson correlation. The performance was measured 130 using three databases of interactors previously benchmarked in Pan et al.: CORUM, a manually 131 curated protein complex database (Ruepp et al., 2008); hu.MAP, a database of protein-protein 132 interactions detected by mass spectrometry experiments (Drew et al., 2017); and STRING, a 133 database of co-functional interactions integrating multiple sources of direct and indirect evidence 134 (Szklarczyk et al., 2017). We found that GLS consistently prioritized genes more effectively than 135 several other methods, including Pearson correlation bias-corrected with PCA using olfactory 136 receptor genes as a gold-standard negative set (Boyle et al., 2018), across all three databases 137 and across a wide variety of rank thresholds (Figure 2A). For instance, the top-ranked partners 138 for each gene are approximately 160-fold enriched for CORUM interactions for GLS compared to 139 120-fold for bias-corrected Pearson correlation; for hu.MAP, 130-fold versus 90-fold enriched; 140 and for STRING, 7.5-fold versus 5.5-fold enriched. Remarkably, failing to perform PCA-based 141 bias correction significantly degrades the performance of Pearson correlation but not GLS, 142 suggesting that GLS is able to automatically perform bias correction without requiring a putatively 143 non-essential gene set like olfactory receptors.

145 We also compared co-essentiality to co-expression, a complementary approach to assessing co146 functionality, using the COXPRESdb database (Okamura et al., 2015). We observed that co147 essentiality substantially outperformed co-expression in recall of protein complexes and physical 148 interactions recorded in the CORUM and hu.MAP databases, but performance was more 149 equivocal for STRING (Figure 2A), with co-essentiality outperforming co-expression only for top150 ranked partner genes. Of note, STRING integrates seven sources of evidence (experimental 
151 evidence, other pathway/complex databases, co-expression, literature text-mining, genomic co-

152 localization across species, co-occurrence across species, and existence of a gene-gene fusion

153 in any species); to reduce the potential for circularity, we restricted to gene pairs supported by

154 experimental evidence. Collectively, these results suggest that co-essentiality and co-expression

155 may have complementary roles in biological pathway mapping, with co-essentiality being better-

156 suited for detecting protein complexes and direct physical interactions and co-expression being

157 better-suited for detecting more indirect functional relationships such as regulatory relationships.

159 Co-essentiality was particularly effective in detecting interactions for a number of key cancer

160 drivers. For example, 8 of TP53's 10 significant co-essential partners are known interactors

161 (USP28, CDKN1A, TP53BP1, MDM2, CHEK2, ATM, PPM1D, UBE2K) compared with only 3 of

162 the top 10 co-expressed partners in COXPRESdb (Table S2). For KRAS, 3 of 5 significant co-

163 essential partners are known interactors compared to none of the top 5 co-expressed partners;

164 and for BRCA1, 3 of 6 co-essential partners are known interactors compared to 1 of 6 for co165 expression.

167 Co-essential modules recapitulate known pathways and nominate novel members

168 To group genes into modules based on their co-essentiality profiles from GLS, we used 169 ClusterONE (Nepusz et al., 2012), a commonly-used algorithm originally developed for the de 170 novo discovery of protein complexes from protein-protein interaction data (see Methods). 171 Crucially, the modules generated by ClusterONE are allowed to be overlapping, enabling 172 pleiotropic genes to be constituents of multiple modules. One major parameter that affects the 173 quality of ClusterONE module detection is the module density $d$, which determines (on a 0-to-1 174 scale) how strong the internal connections within a cluster must be relative to the connections on

175 the edge of the cluster between members and non-members. It has previously been observed 176 that inferring networks at multiple scales helps provide the most complete picture of biological 
177 systems (Dutkowski et al., 2013; Kramer et al., 2014); accordingly, we found that lower values of $178 d$ (e.g. $d=0.2$ ) led to larger modules and better performance on STRING, while larger values 179 (e.g. $d=0.9)$ led to smaller modules and better performance at recapitulating complexes and 180 physical interactions from CORUM and hu.MAP, with intermediate values (e.g. $d=0.5)$ striking a 181 balance between the two (Figure S3). Because modules generated with different values of $d$ 182 capture different types of biological pathways, we generated a combined list of modules using $d$ $183=0.2(\mathrm{~N}=168), d=0.5(\mathrm{~N}=1892)$ and $d=0.9(\mathrm{~N}=3169)$ (Table S3).

185 The 5,218 co-essential modules in this almanac, containing between 4 and 741 genes, 186 correspond to a wide range of biological pathways (Table S3). To estimate the fraction of the genome our modules assign a putative function, we counted the number of genes included in a module that is highly (at least 100-fold) enriched for some GO term. By this metric, our set of coessential modules assign putative functions to 14,383 genes, a much larger fraction of the genome compared to previous approaches used to cluster genes based on co-essentiality profiles

191 (Figure 2B).

Among the 1,311 modules with greater than 100-fold enrichments are modules highly enriched

194 for genes involved in growth regulation (Figures 3A, B), autophagy (Figure 3C), cell-cell signaling 195 (Figure 3D), the DNA damage response (Figure 3E), innate immunity (Figure 3F), glycolysis 196 (Figure 3G), transcriptional regulation (Figures $\mathbf{3 H}$, I), the cell cycle (Figure $\mathbf{3} \mathbf{J}$ ), and 197 mitochondrial respiration (Figure 3K), among many others (Table S3).

199 Several important features of the co-essential modules are highlighted by the examples shown in

200 Figure 3. First, the ability of ClusterONE to include genes in multiple modules enabled 201 identification of pleiotropic gene functions, as illustrated by the identification of two modules 202 containing MTOR that closely correspond to the two mTOR-containing complexes, mTORC1 
(Figure 3A) and mTORC2 (Figure 3B) (Saxton and Sabatini, 2017). Second, co-essential modules are not limited to physical complexes, as illustrated by the near-complete identification of the glycolysis pathway (Figure 3G), or even to cell-autonomous pathways, as illustrated by the identification of the Jagged-Notch intercellular signaling pathway (Figure 3D). Third, by examining modules identified at different values of $d$, we were able to detect multiple scales of biological organization, as illustrated by the set of modules we identified that correspond to mitochondrial respiration (Figure 3Ki-v). Module \#256, a 163-member module identified at $d=0.2$, includes

210 most nuclear-encoded subunits of the four respiratory chain complexes required for mitochondrial 211 ATP synthesis, as well as numerous mitochondrial tRNA synthetases, elongation factors, and 212 components of the mitoribosome required for synthesis of the mitochondrial subunits of the 213 mitochondrial respiratory complexes (Figure 3K). Several modules identified with $d$ set to 0.9 , by contrast, correspond to smaller units of functional organization, such as module \#4250, a 13-

215 member module that contains 12 subunits of the ATP synthase complex (Figure 3Kiv, Table S3), 216 and module \#2072, a 99-member module comprising 61 subunits of the mitochondrial ribosome 217 and many of its associated factors (Figure 3Kv, Table S3). Fourth, we noted that whereas several 218 modules are nearly "complete" representations of a biological pathway, such as module \#520, 219 which comprises most of the genes identified in recent targeted screens for autophagy regulators

220 (Figure 3C, cf. Shoemaker et al., 2019), and no genes not previously implicated in autophagy, 221 many modules highly enriched for a particular pathway also contain one or more uncharacterized 222 genes (red boxes, Figure 3E, F, I, H, J, K).

224 Using co-essential modules to systematically predict the functions of uncharacterized 225 genes

226 Hundreds of human genes have not been assigned any molecular function. Co-essentiality 227 profiling has recently been used to assign uncharacterized genes to pathways, with predictions 228 based on the functions of the genes that have the largest Pearson correlations with the 
uncharacterized gene (Pan et al., 2018; Wang et al., 2017). However, it has remained unclear how broadly useful co-essentiality information is in predicting the functions of the hundreds of genes that remain uncharacterized, which likely span diverse biological processes.

Co-essential modules are in many cases highly enriched for functionally related genes, and thus enable unbiased, genome-wide prediction of uncharacterized gene function. To generate a list of

235 functional predictions for uncharacterized genes, we first mined the UniProt database to assemble

236 a list of uncharacterized genes, which we defined as those genes with UniProt annotation score

237 (a heuristic measure of protein annotation content) of 2 or lower. We then enumerated all the 238 uncharacterized genes present in modules at least 100-fold enriched for one or more GO terms, 239 excluding GO terms with $<5$ genes.

241 The 102 uncharacterized genes assigned putative functions by this method are included, on

242 average, in $\sim 2$ co-essential modules, yielding a list of 220 functional predictions (Table S4). We

243 excluded uncharacterized genes in syntenic modules (i.e. modules comprising genes all located

244 on the same chromosome) from this count, since while many syntenic modules likely represent

245 bona fide co-functional gene sets, others may be confounded by residual copy number artifacts

246 or other factors (Methods). Notably, several of these predictions are consistent with recent

247 experimental information that has not yet been incorporated into the Uniprot database, including

248 C19orf52 in mitochondrial import (Kang et al., 2016), C16orf59 in centriole function (Breslow et

249 al., 2018), TMEM261 in mitochondrial respiratory complex I (Stroud et al., 2016), and PTAR1 in

250 Golgi function (Blomen et al., 2015), showcasing the power of this method in assigning gene

251 functions. To prioritize a list of functional predictions for experimental validation, we ranked

252 modules by their maximal enrichment for a given GO term, because these predictions yield the

253 most readily testable predictions. The top uncharacterized gene predictions (ranked by GO term 
254 enrichment) span a wide range of biological processes, including mitochondrial respiration,

255 transcription, DNA repair, Golgi function, lipid synthesis, and endocytosis (Table S4).

TMEM189 encodes the orphan enzyme plasmanylethanolamine desaturase required for plasmalogen synthesis

We selected two genes, TMEM189 (ranked \#1) and C15orf57 (ranked \#18), for experimental validation. TMEM189, also known as $K U A$, encodes a 270 amino acid transmembrane protein whose function was largely unexplored prior to our work, with previous studies focused on the observation that it can be transcribed as both an independent ORF and as a fusion with the neighboring UBE2V1 gene. Both TMEM189 and TMEM189-UBE2V1 have been observed to localize to the endoplasmic reticulum (Thomson et al., 2000).

The top-ranked co-essential module containing TMEM189, module 2213 , is highly enriched for genes required for synthesis of ether lipids (Figure 4A), which comprise a broad class of structural and signaling lipids involved in regulation of membrane fluidity and sensitivity to oxidative stress, and which account for approximately $20 \%$ of the phospholipids in human cells (Nagan and Zoeller, 2001). We noted that genes in this module appeared to be particularly essential in cell lines

271 derived from haematological cancers (Figure 4B). Whereas several genes in this module,

272 including AGPS, FAR1, and GNPAT, are specifically involved in ether lipid synthesis, some genes

273 contained in this module, including PCYT2 and EPT1, are required for both ether lipid synthesis

274 and the synthesis of other ethanolamine-containing phospholipids. Based on this prediction, we

275 hypothesized that TMEM189 could play a role in lipid biosynthesis and have a specialized role in

276 the synthesis of ether lipids.

278 To interrogate the functional role of TMEM189 in lipid biosynthesis in an unbiased manner, we 279 extended a targeted lipidomics method (Contrepois et al., 2018; Schüssler-Fiorenza Rose et al., 
2019) to measure the absolute concentrations of several hundred lipid species in cell extracts.

281 We compared concentrations of these lipids in cell extracts derived from HeLa-Cas9 cells that

282 stably expressed single guide RNAs (sgRNAs) targeting either TMEM189 or a control genomic

283 locus. Strikingly, though the vast majority of quantified lipid species were present in similar

284 concentrations in both types of cell extracts, cell extracts expressing TMEM189-targeting sgRNAs

285 contained dramatically lower concentrations of 37 lipid species belonging to the ether lipid

286 subclass plasmenylethanolamines (Figure 4C, 4D, Table S5), also known as ethanolamine

287 plasmalogens, and higher concentrations of 30 lipid species belonging to ether lipid subclass

288 plasmanylethanolamines (Figure 4C, 4E). Plasmanylethanolamines differ from

289 plasmenylethanolamines in the presence of a single double bond in the sn-1 acyl chain, which

290 forms part of the plasmalogen-defining vinyl ether bond. Plasmanylethanolamines and

291 plasmenylethanolamines form a known precursor-product relationship, with

292 plasmanylethanolamines rapidly converted into plasmenylethanolamines in the endoplasmic

293 reticulum by the orphan enzyme plasmanylethanolamine desaturase (PEDS), which was first

294 reported in mammalian cell extracts over forty years ago (reviewed in (Snyder et al., 1985)).

296 The accumulation of the precursors, and loss of the product, of the reaction catalyzed by PEDS 297 in cells expressing TMEM189-targeting sgRNAs strongly implicates TMEM189 as the gene 298 responsible for orphan PEDS activity. Two orthogonal lines of evidence strongly support this 299 conclusion. First, we examined a cell line, RAW.12, that was evolved to lack plasmalogens and 300 shown to exhibit a specific defect in PEDS activity (Zoeller et al., 1992), and determined whether 301 this cell line exhibits deficient expression of TMEM189. By immunoblotting for TMEM189 in cell 302 extracts prepared from RAW.12 cells or its parent, unmutated cell line, RAW264.7, we confirmed 303 that TMEM189 levels were decreased in PEDS-deficient RAW.12 cell extracts (Figure 4F). 304 Second, TMEM189 bears sequence features consistent with a function in lipid desaturation. 305 TMEM189 contains a histidine-rich domain conserved in most lipid desaturase enzymes, and is 
distantly related to the fatty acid desaturase FAD4 in Arabidopsis (Gao et al., 2009), which introduces an unusual double bond in the sn-2 fatty acid (Gao et al., 2009).

We noted that TMEM189 is also present in a co-essential module, module \#808, which is highly enriched for genes involved in the biosynthesis of sphingolipids, a distinct class of lipids that

311 predominantly localizes to the plasma membrane and, similarly to ether lipids, contributes to both

312 signaling and membrane structure and fluidity. In lipidomic analyses, cell extracts derived from

313 cells expressing sgRNAs targeting SPTLC2, a subunit of serine palmitoyltransferase, the rate

314 limiting enzyme in sphingolipid biosynthesis, were highly depleted of several sphingolipid species,

315 whereas abundances of most sphingolipid species were largely unaltered in cell extracts from

316 HeLa cells expressing TMEM189-targeting sgRNAs, ruling out a central role for TMEM189 in 317 sphingolipid biosynthesis (Table S5). However, we observed that the relative abundances of

318 several very long chain sphingomyelin species were altered in cells lacking TMEM189, with

319 sphingomyelins with C26 fatty-acids decreased in abundance and C22 and C24 sphingomyelins

320 increased in abundance (Figure S4A, Table S5). We additionally found that affinity-purified

321 TMEM189-GFP complexes, isolated from HeLa cells, were highly enriched for SPTLC2 (Figure

322 S4B, Table S6). Further work is required to determine whether this pair of observations - that

323 TMEM189 and SPTLC2 appear to physically interact, and that the abundances of very long chain

324 sphingomyelin species are subtly altered in TMEM189-knockout cells - reflects a direct role for

325 TMEM189 in the regulation of fatty acid incorporation into ceramides. Alternatively, because

326 sphingolipid composition is tightly regulated to maintain membrane fluidity (Breslow and

327 Weissman, 2010), the altered sphingolipid profile observed in TMEM189-knockout cells may

328 reflect a compensatory response to loss of plasmalogens and resulting disrupted membrane

329 composition in TMEM189-knockout cells. Regardless of these possibilities, our results provide

330 conclusive evidence for a primary role for TMEM189 as the orphan desaturase required for the 
331 final step of plasmalogen biosynthesis and provide a striking example of the power of co-essential 332 modules to predict gene function.

\section{C15orf57 is a novel regulator of clathrin-mediated endocytosis}

335 C15orf57 (also known as coiled-coil domain containing 32 (CCDC32) encodes a 185-residue

336 protein with no annotated function. Recent reports described the existence of a chimeric transcript

337 of unknown significance that contains $C 15$ orf57 and the gene CBX3 in certain tumor samples (Xu

338 et al., 2014). C15orf57 is present in several overlapping co-essential modules (Table S3),

339 including a module (\#2067) that is highly enriched for genes required for clathrin-mediated

340 endocytosis, in particular subunits of the adaptor protein 2 (AP2) complex (Figure 5A, B). One of

341 the best-described functions of the AP2 complex is to mediate endocytosis of transferrin bound

342 to the transferrin receptor (Motley et al., 2003), so we hypothesized that C15orf57 might be

343 required for cellular uptake of transferrin. To test this, we monitored uptake of transferrin, labeled

344 with a pH-sensitive fluorescent dye, pHrodo, by HeLa-Cas9 cells expressing sgRNAs targeting

345 either C15orf57, the transferrin receptor (TFRC), or a control locus. Cells expressing sgRNAs

346 targeting either C15orf57 or TRFC exhibited reduced uptake of transferrin compared to cells

347 expressing control sgRNAs, consistent with a role for C15orf57 in transferrin uptake (Figure 5C).

349 To gain further insight into the mechanism by which C15orf57 functions in clathrin-mediated 350 endocytosis, we immunoprecipitated C15orf57-GFP complexes and analyzed them by mass 351 spectrometry. C15orf57-GFP immunoprecipitates were strongly enriched for all five members of 352 the AP2 clathrin adaptor complex: AP2S1, AP2A1, AP2A2, AP2M1, and AP2B1 (Figure 5D, 353 Table S6). In reciprocal co-immunoprecipitation experiments, we confirmed that C15orf57-GFP 354 physically interacts with AP2S1-mCherry (Figure 5E). We additionally confirmed through confocal 355 microscopy that C15orf57-GFP colocalizes with AP2S1-mCherry in small puncta at the cell 356 surface that likely correspond to clathrin-coated pits, the sites of clathrin-mediated endocytosis 
357 (Figure 5F). The identification of the members of the AP2 complex as physical interactors of 358 C15orf57, and their colocalization in cells, suggests that C15orf57 may regulate clathrin-mediated 359 endocytosis of transferrin (and possibly other cargoes) by directly modulating AP2 function.

361 Identification of cancer type-specific pathway dependencies

362 A major motivation for high-throughput cancer cell line screening efforts, such as the Achilles 363 project underlying this work, is the possibility of identifying cancer type-specific vulnerabilities that 364 could be exploited as therapeutic targets (Tsherniak et al., 2017; Wang et al., 2017). These efforts 365 have shown promise in identifying individual genes that are selectively essential in specific cancer 366 types (Chan et al., 2019; Wang et al., 2017). Some cancers have also been observed to harbor 367 selective dependencies on entire gene pathways (Hart et al., 2015, Campbell et al., 2016). We 368 asked whether our list of co-essential modules, many of which are highly enriched for genes that 369 function in the same pathway, could be used to identify cancer-type specific pathway 370 dependencies.

372 To systematically identify differentially-essential modules across tissue types, we obtained cancer 373 type-specific pathway dependency $p$-values for each module-cancer type pair by first calculating

$374 p$-values for each gene and then aggregating $p$-values across genes in each module. To obtain 375 uninflated $p$-values, we again applied GLS (see Methods). Using this conservative approach, we 376 identified 444 modules that are differentially essential in cancers derived from 16 distinct tissue 377 types (Figure 6A, Table S7).

379 Several of the modules that are most differentially essential in specific tissue types correspond to 380 canonical tissue-specific cancer drivers, demonstrating the power of this approach to uncover 381 bona fide selective pathway dependencies. As one example, the most significantly breast cancer382 specific module dependency contains ESR1, the estrogen receptor (ER), which is overexpressed 
in over $70 \%$ of breast cancers and enables hormone-dependent growth (Ariazi et al., 2006). This module (as well as the neighborhood that corresponds most closely to this module in the twodimensional representation of the network, Figure 6B) also contains several genes that functionally interact with ESR1, including SPDEF, FOXA1, and GATA3, three master regulators of estrogen-receptor-dependent gene expression in breast cancer (Fletcher et al., 2013); retinoic acid receptor alpha $(R A R A)$, a target of ESR1-dependent transcriptional activity (Roman et al., 1993); and TOB1, a gene required for estrogen-independent growth of ER-positive breast cancers

390 (Zhang et al., 2016).

As a second example, the most significantly differentially-essential module in skin cancer (and its corresponding neighborhood) (Figure 6C) includes several components of the BRAF-MAPK pathway, which is consistent with the fact that $B R A F$ is mutated in $\sim 50 \%$ of melanomas (Ascierto et al., 2012), as well as MITF, a melanoma-specific oncogene (Garraway et al., 2005) activated downstream of BRAF. Additional module members, including NFATC2, SOX9, and SOX10, have well-established roles in melanoma (Harris et al., 2010; Perotti et al., 2016). In both of these examples, the co-essential modules we identified as selectively required in certain cancer types contain sets of lineage-specific cancer drivers that are known to functionally interact, illustrating the power of our approach in identifying cancer pathway dependencies. The additional 442 modules that we identify as selectively essential in 16 cancer types (Table S7) represent a resource for identifying novel pathway targets in specific cancer types.

\section{An interactive resource for biological discovery}

405 We created a web tool (coessentiality.net) (Figure S5; Video S1) to enable dynamic visualization 406 and exploration of the genome-wide co-essentiality map shown in Figure 1C, for which we 407 anticipate several distinct uses. First, this tool can be used as a starting point for gaining insight 408 into the function of any gene: users can search for a gene of interest, for example, KRAS (Figure 
S5, Video S1), which is then highlighted on the interactive 2D layout in the context of its neighborhood. To understand relationships between sets of genes in a given neighborhood, users

411 can then directly select gene neighborhoods with the cursor (Video S1). Once a gene set is 412 selected, two data panels are generated: a biclustered heatmap of the genes' essentiality profiles 413 across the 485 cell lines and a plot of the gene set's enrichment for annotated pathways, 414 complexes and gene ontology terms (Ashburner et al., 2000; The Gene Ontology Consortium, 415 2017) (Figure S5, Video S1). Users can compare the essentiality profile of the selected gene set 416 with the mutational status and expression level of other selected genes. For example, with the 417 KRAS-containing neighborhood selected, users can plot the lines in which KRAS is mutated, 418 which reveals that KRAS and several genes in its neighborhood are selectively essential in KRAS419 mutated lines (Figure S5, Video S1). Users can also gain insight into pathways that are 420 particularly required for the growth of individual cancer lines or for cancers derived from a certain 421 tissue by selecting cell lines (for example, U937 cells, Video S1) or tissue types (for example, 422 kidney cancers, Figure S5, Video S1) from drop-down menus, causing the two-dimensional 423 network to be colored according to each gene's essentiality in the selected cell line or tissue. 424 Finally, users can upload a specified set of genes - for example, the members of the endocytosis 425 module containing C15orf57 (Figure 5A, Video S1) - to understand relationships between 426 multiple genes of interest. We anticipate this tool will be a broadly useful starting point for the 427 functional characterization of genes and gene sets as well as a powerful hypothesis-generating 428 platform for users interested in identifying cancer-type specific pathway dependencies. 


\section{DISCUSSION}

430 Building a global map of biological pathways in human cells and assigning function to the

431 thousands of poorly characterized genes remain key challenges in cell biology. In this work, we

432 demonstrate that mapping co-essentiality across a diverse spectrum of cancer cell lines enables

433 significant progress toward both objectives.

435 The co-essential network developed here represents, to our knowledge, the most comprehensive 436 and statistically robust genome-wide perturbational pathway map of human cells to date. Unlike 437 double-perturbation approaches, our approach is scalable to all pairs of genes in the genome; 438 and unlike prior approaches to co-essentiality mapping, it is statistically well-calibrated despite 439 the lack of independence among the screens it was derived from. A recent comparison of modules 440 derived from different biological networks suggested that modules created based on co441 expression data are better able to recall gene relationships than co-essentiality data, in a GWAS442 based benchmarking approach (Choobdar et al., 2019). By contrast, we find that co-essentiality443 derived networks outperform co-expression-derived networks in their ability to recall protein 444 complexes. The gene-gene relationships evidenced by these different datasets may be 445 complementary, with co-essentiality especially well-powered to detect protein complexes and co446 expression better able to detect certain indirect pathway relationships (Figure 2A). Our global

447 interaction map and associated web tool showcase the high resolution and versatility of co448 essentiality as a method for de novo pathway mapping.

450 Our validations of the role of TMEM189 in plasmalogen biosynthesis and C15orf57 in clathrin451 mediated endocytosis highlight the utility of biological hypothesis generation from co-essential 452 modules. Of note, during the preparation of this manuscript, an entirely orthogonal approach 453 based on the study of the bacterial protein CarF, a homolog of TMEM189, revealed that this 454 enzyme is responsible for PEDS activity in bacterial cells, and this activity was shown to be 
conserved in human cells (Gallego-García et al., 2019). The complementary approaches and orthogonal validations of TMEM189 as the key enzyme for plasmalogen synthesis will potentiate

457 dissection of the functions of this largely understudied class of lipids. The specific function of the 458 plasmalogen-defining vinyl ether bond, which has been proposed to be critical for antioxidant and 459 oxygen-sensing functions of plasmalogens, has remained difficult to assess experimentally. With 460 the identity of plasmanylethanolamine desaturase now in hand, these and other basic questions 461 about plasmalogen function can be addressed. Plasmalogens have been noted to be highly 462 upregulated in a variety of malignancies, and inhibitors of this pathway have recently been 463 explored as anti-cancer agents (Piano et al., 2015). With the discovery of TMEM189 as a novel 464 enzyme required for plasmalogen synthesis, we uncover an additional therapeutically targetable 465 node in this pathway.

467 Our identification of C15orf57 as a regulator of clathrin-mediated endocytosis adds another key 468 player to this pathway; further work is required to uncover its precise mechanistic function. 469 Nonetheless, the role of C15orf57 in binding the AP2 complex and regulating endocytosis that we 470 describe here may advance understanding of the significance of recurrent $C 150 r f 57-C B X 3$ gene 471 fusions that have been proposed to contribute to hepatocellular carcinoma (Zhu et al., 2019). In 472 addition to the two uncharacterized genes for which we experimentally validated their predicted 473 functions, we note that several additional functional predictions generated by our method are 474 supported by evidence from other unbiased, high-throughput approaches. For example, C7orf26, 475 which we predict is involved in the function of the integrator complex that is required for 476 transcription of small non-coding RNAs (Chen and Wagner, 2010) was observed to interact with 477 several subunits of the integrator complex in high-throughput IP-MS experiments; its expression 478 is also highly correlated with several integrator subunits (Okamura et al., 2015; Szklarczyk et al., 479 2017). As a second example, the functionally uncharacterized gene TMEM242, for which we 480 predict a function in mitochondrial respiration, was reported to interact with the gene product of 
NDUFA3, a subunit of mitochondrial complex I, in a high-throughput study (Szklarczyk et al., 2017). Overall, our experimental validation of two uncharacterized gene predictions, paired with our list of 100 additional uncharacterized genes for which we predict a function, provides an

484 immediately useful resource for the broader cell biology community.

Beyond nominating functions for entirely uncharacterized genes, the modules identified in this study have the potential to suggest novel roles for genes with existing functional annotations. Within the modules that were the focus of experimental validations in this study, we note that SEC14L1, previously characterized for its role in inhibiting the anti-viral RIG-1 pathway (Li et al., 2013), is now the only gene in 8-gene module \#2213 that has not been shown to be required for ether lipid synthesis. Notably, SEC14L1 contains a conserved lipid-binding domain, and is related to a yeast gene, SEC14, involved in non-vesicular lipid transport (Saito et al., 2007). Plasmalogens are known to traffic to the cell surface after being synthesized in the endoplasmic

494 reticulum, but the factors that regulate plasmalogen trafficking, and the route plasmalogens take 495 to the plasma membrane, remain undefined; the possibility that SEC14L1 regulates either 496 plasmalogen synthesis or transport is thus a prime example of an experimentally testable 497 hypothesis motivated by our findings. Indeed, preliminary support for this hypothesis is provided 498 by in vitro studies of yeast Sec14, which revealed that purified Sec14 is sufficient to catalyze ether 499 lipid transport between lipid membranes (Szolderits et al., 1991). Further study is required to 500 confirm that human SEC14L1 can similarly drive ether lipid transport between membranes and to 501 discern whether non-vesciular lipid transport by SEC14L1 could mediate ether lipid trafficking to 502 the cell surface in living cells.

503

504 An additional key feature of the co-essential modules we identify, by virtue of their overlapping 505 nature, is their ability to recapitulate multiple levels of biological organization as well as 506 relationships between distinct pathways and complexes, as exemplified by the set of modules 
corresponding to distinct complexes of the mitochondrial respiratory chain (Figure $3 \mathbf{K}$ ). The set of modules enriched for the 10-subunit endoplasmic reticulum (ER) membrane complex (EMC) provides an additional striking example. The EMC complex was recently shown to function as a

510 transmembrane insertase required for the biogenesis of a subset of transmembrane proteins, 511 particularly tail-anchored and polytopic proteins (Guna et al., 2018, Shurtleff et al., 2018) and is 512 additionally noted for its role in cholesterol homeostasis (Volkmar et al., 2019). We identify several 513 modules enriched for EMC subunits, including a 7-gene module (\#5037) containing 6 EMC

514 subunits and TMEM147, a gene recently shown to cooperate with EMC in transmembrane protein

515 biogenesis (Talbot et al., 2019), and a 16-gene module (\#2450) containing 8 EMC subunits and

5162 genes (MBTPS1 and SCAP) required for cholesterol homeostasis. In addition, we identify a 23-

517 gene module (\#534) that contains 5 EMC subunits and 12 subunits of the lysosomal V-ATPase

518 complex (8 of which are separately contained in 9-gene module 2450). Several of these V-

519 ATPase subunits were recently identified in an unbiased proteomic study as among the proteins

520 most dependent on EMC function for their stability (Tian et al., 2019). Thus, the set of modules

521 we identify that are enriched for EMC subunits correspond to known inter-pathway interactions,

522 and demonstrate the power of co-essential modules to not only identify individual pathways but

523 to point to possible inter-pathway relationships.

525 Numerous additional modules (Figure 3B, D, E, F, J, K, Table S3) that are highly enriched for 526 one GO term contain components of additional pathways not previously linked to the most527 enriched pathway. As one example, two essential components of the mitotic spindle checkpoint, $528 B U B 1 B$ and MAD2L1, are present not only in a module (\#1360) highly enriched for genes involved 529 in kinetochore/spindle checkpoint function but also, unexpectedly, in a module (\#739) highly 530 enriched for interferon (IFN) response genes (Figure 3F). BUB1B and MAD2L1 have well-defined 531 roles in the prevention of chromosome instability (CIN) (Ricke et al., 2008) but have not previously 532 been linked to interferon gene function. One possible connection is suggested by the recent 
533 observation that tumor cells with high levels of CIN generate cytosolic DNA that activates an IFN

534 response and drives cancer progression (Bakhoum et al., 2018). BUB1B and MAD2L1 prevent a

535 particular form of $\mathrm{CIN}$ - aneuploidy resulting from premature sister chromatid separation - but the

536 relevance of this type of CIN in the pathogenic process described in that study was not addressed

537 and may warrant further investigation.

538

539 A key future direction in expanding the ability of this resource to detect functional genetic

540 relationships is to measure additional phenotypes beyond cancer cell line growth under standard

541 conditions. The 485 cell lines screened thus far are derived from a wide range of tissue types and

542 exhibit a highly diverse set of mutational backgrounds, and the Achilles project plans to extend

543 this screening to several thousand cell lines. Nonetheless, our approach has the potential to

544 benefit greatly from screens performed in primary tissues; across individuals; under non-ambient

545 conditions, such as in the presence of a drug or cellular stress; or with readouts other than cellular

546 fitness, such as changes to cell morphology, gene expression, or cellular activity. Such screens

547 offer the potential to uncover an even broader spectrum of functional interactions, and could

548 enable a dynamic map of pathway rewiring across conditions. Overall, our genome-wide mapping

549 of the human co-essential network comprises a powerful resource for biological hypothesis

550 generation and discovery. 


\section{ACKNOWLEDGMENTS}

552 We thank Raphael Zoeller for providing RAW.12 cells and the parent cell line. We gratefully

553 acknowledge Evan Boyle, Justin Donnelly, Mackenzie Pearson, Grace Anderson, Scott Simpkins,

554 Trey Ideker, and members of the Bassik and Kundaje labs for helpful discussions. This work was

555 supported by an NIH Director's New Innovator award (1DP2HD084069-01) to M.C.B., a grant

556 from NIH/ENCODE (5UM1HG009436-02) to A.K. and M.C.B., a Stanford Bio-X Bowes Fellowship

557 to M.W., a Stanford School of Medicine Dean's Postdoctoral Fellowship to R.A.K., and a Jane

558 Coffin Childs Postdoctoral Fellowship to R.A.K.. 
bioRxiv preprint doi: https://doi.org/10.1101/827071; this version posted November 3, 2019. The copyright holder for this preprint (which was not certified by peer review) is the author/funder. All rights reserved. No reuse allowed without permission.

\section{Figure 1}

A

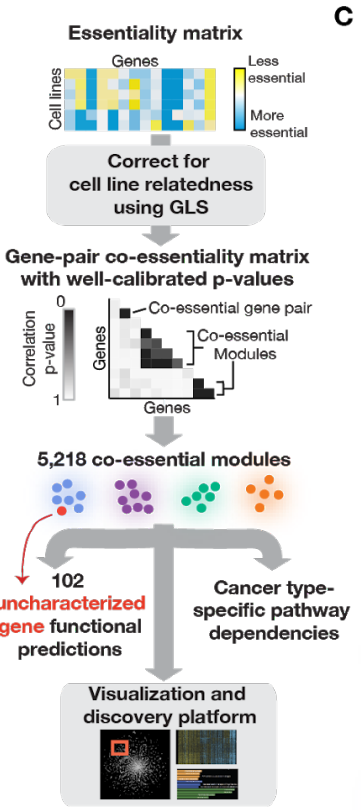

B $\quad \bar{\sigma} 35$. Median $p=0.21$

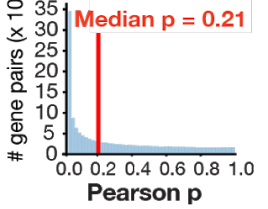

ळ

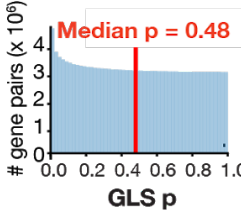

D

mTORC2/PI3K/AKT signaling

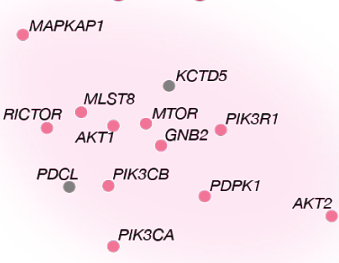

p53 signaling

VEZF1 KHDRBS1

TP53 WWRAP53

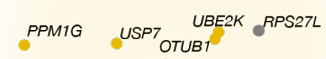

RPL22 BRAPUSP28 MDM2 UBE2D3 ZNF699 USP42-D I, -CDKN1A

CENPF TPBBP' ATM PPM1D

MDM4

C

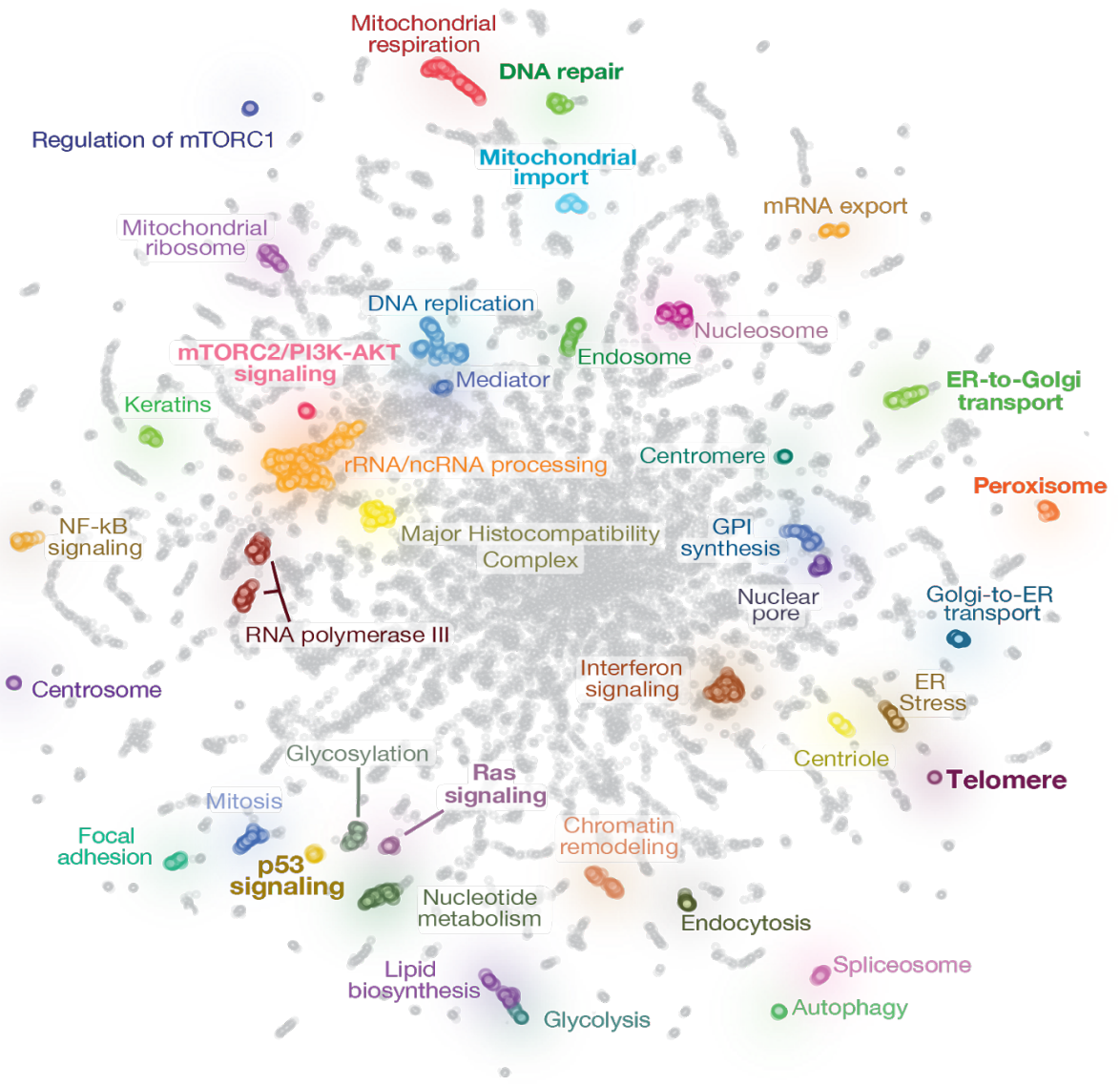

Mitochondrial import

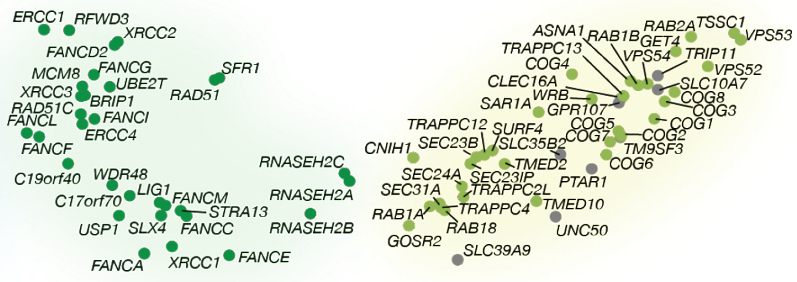

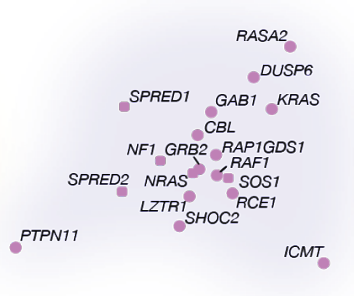

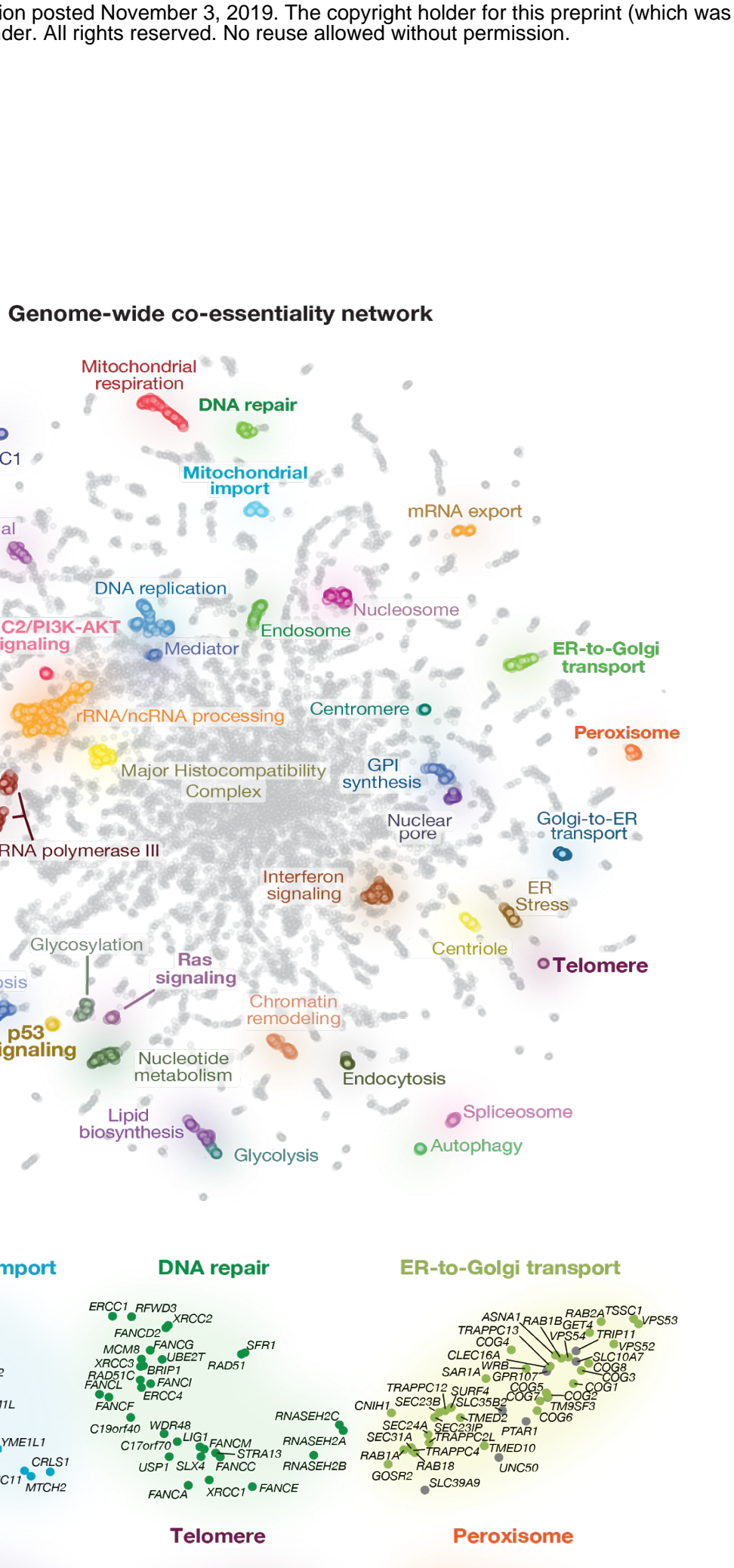

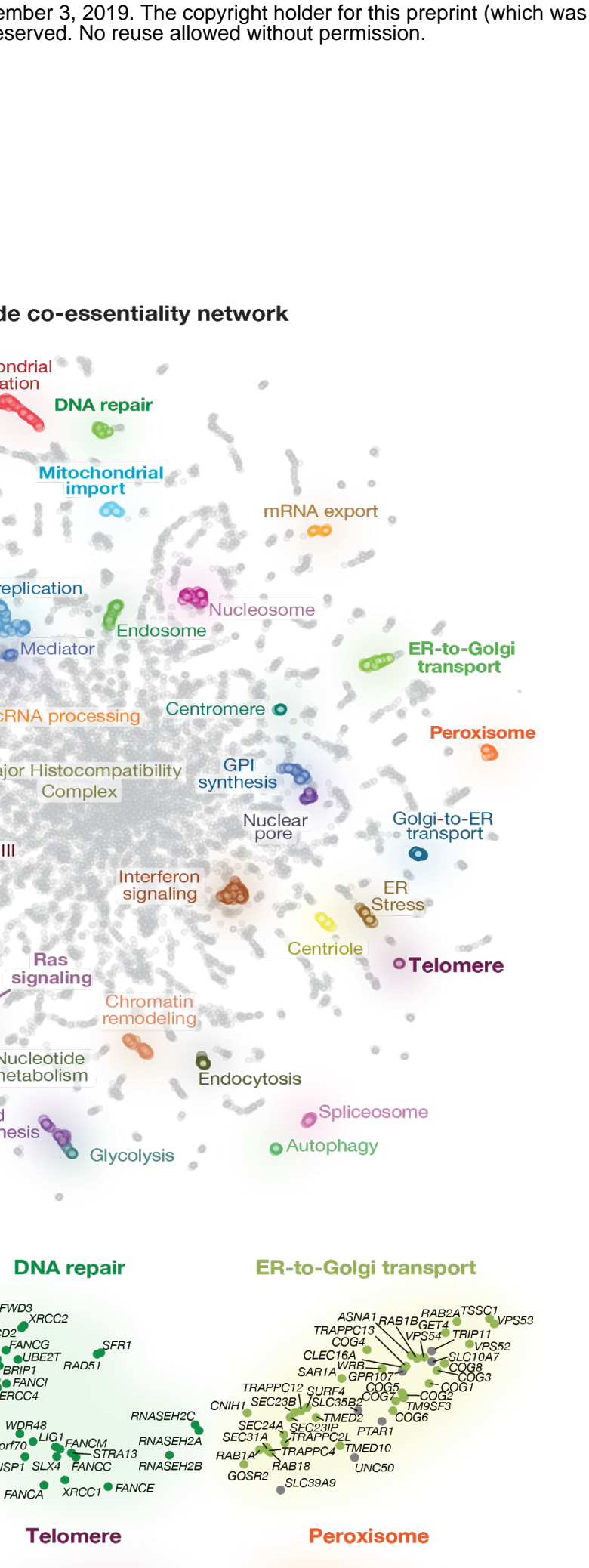

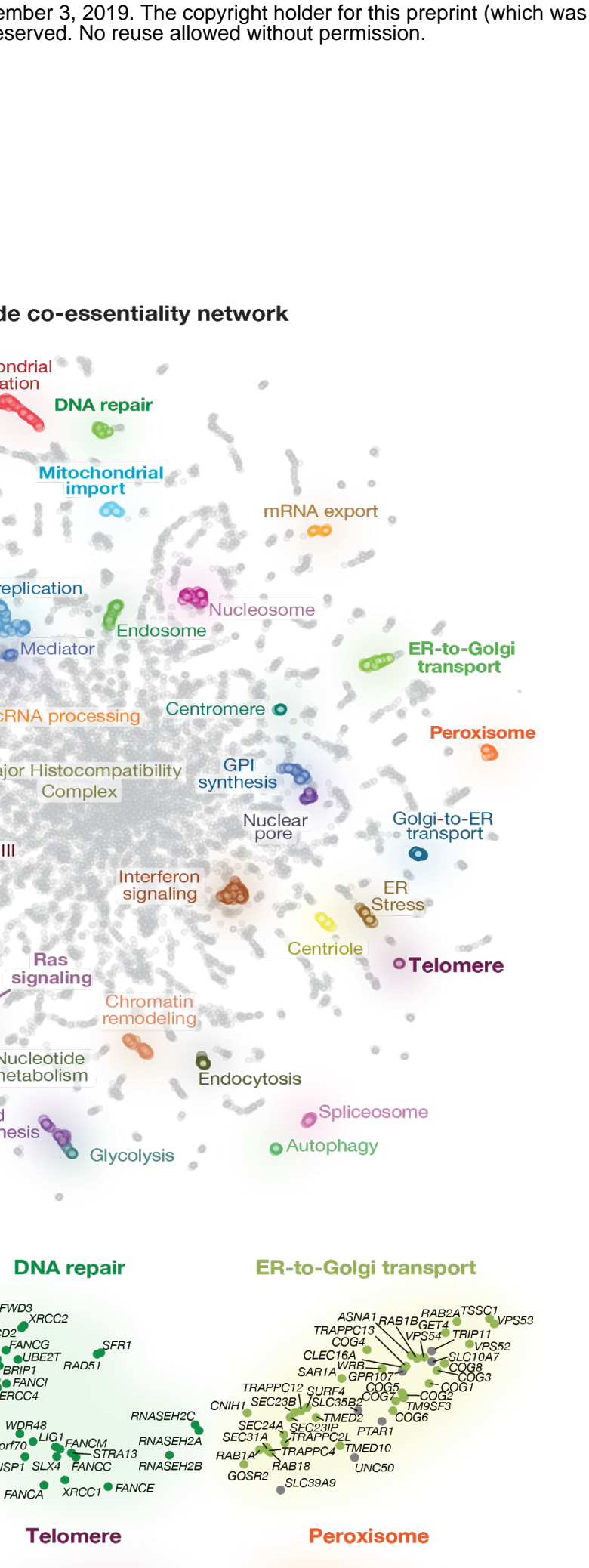

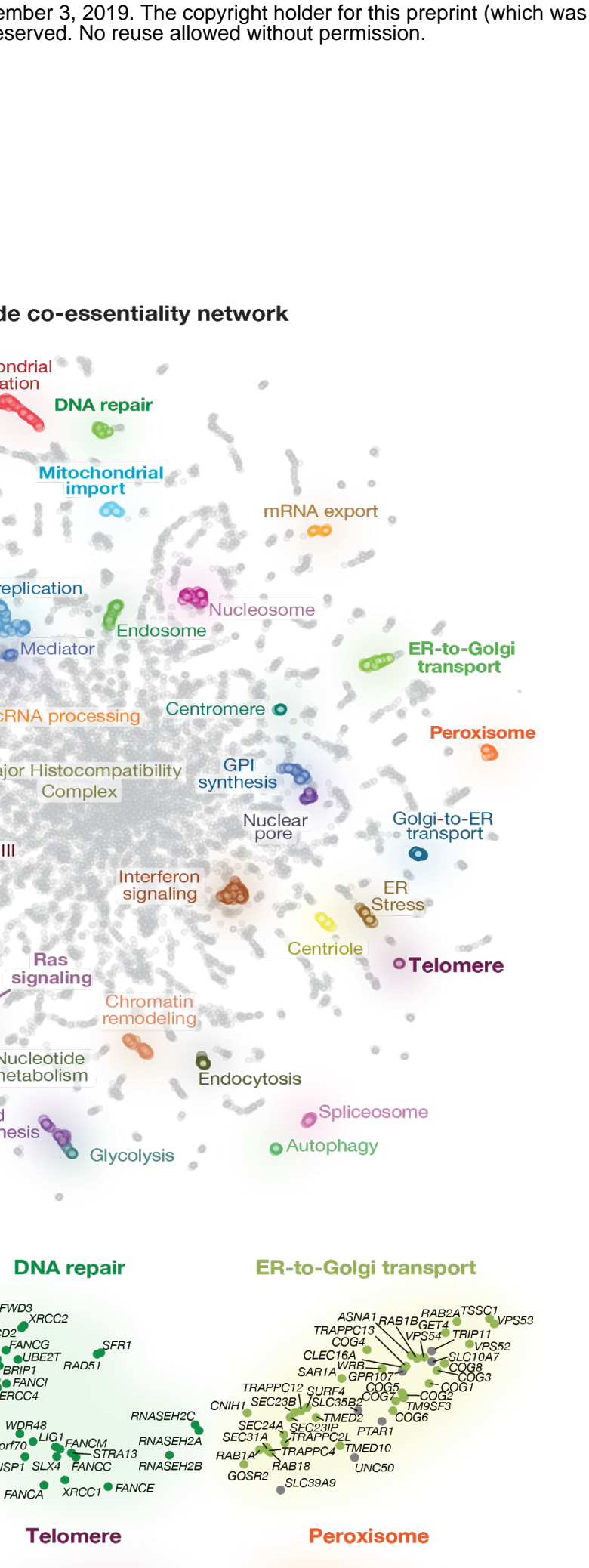

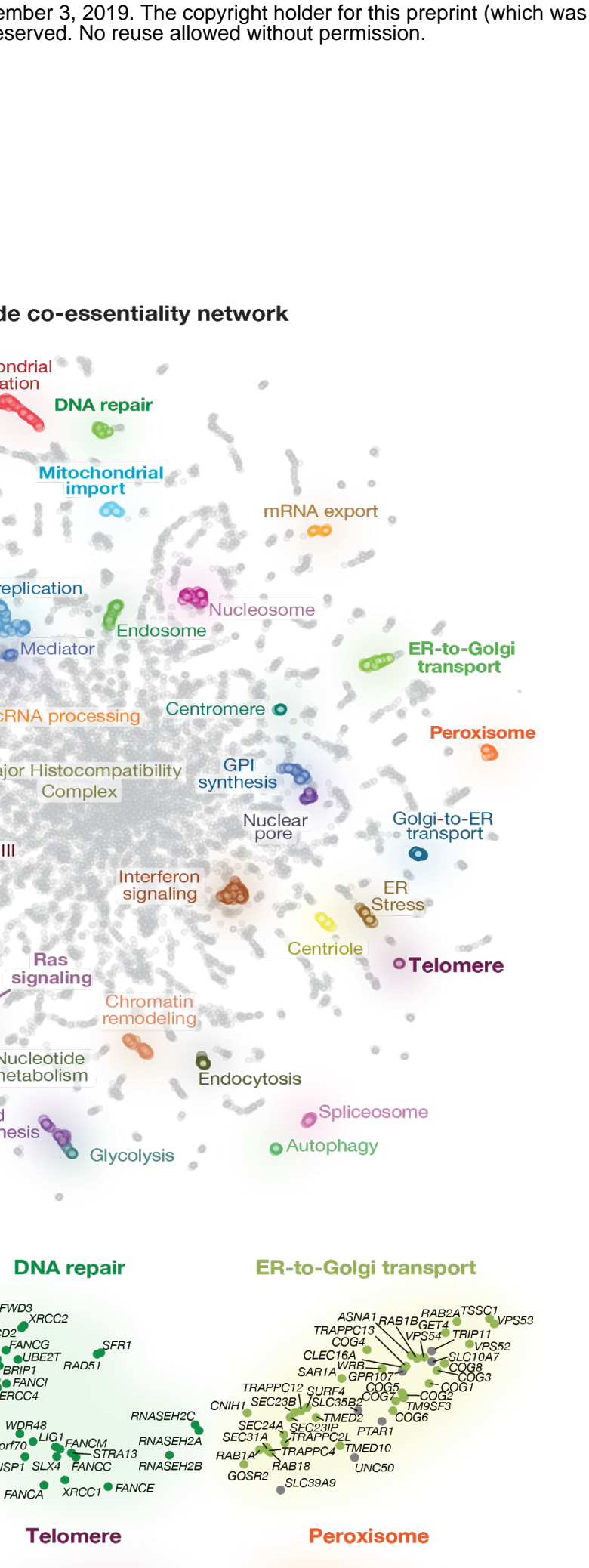

SCP2 PEX2

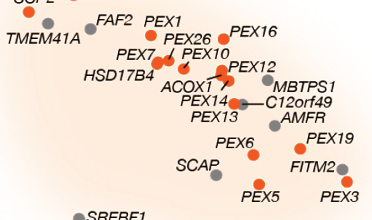

-SREBF1

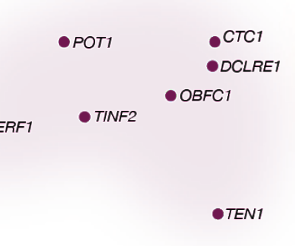


559 Figure 1: Construction of a genome-wide co-essentiality network.

560 (A) Overview of our approach.

561 (B) Histograms of GLS and Pearson correlations across all pairs of genes.

562 (C) Global structure of the co-essentiality network, with manually annotated 'neighborhoods'

563 highly enriched for particular pathways and complexes. Bolded neighborhood labels are

564 highlighted in (D).

565 (D) Selected neighborhoods, with manually-defined known pathway members indicated in color 566 and other genes in gray.

567 See also Figure S1. 


\section{Figure 2}
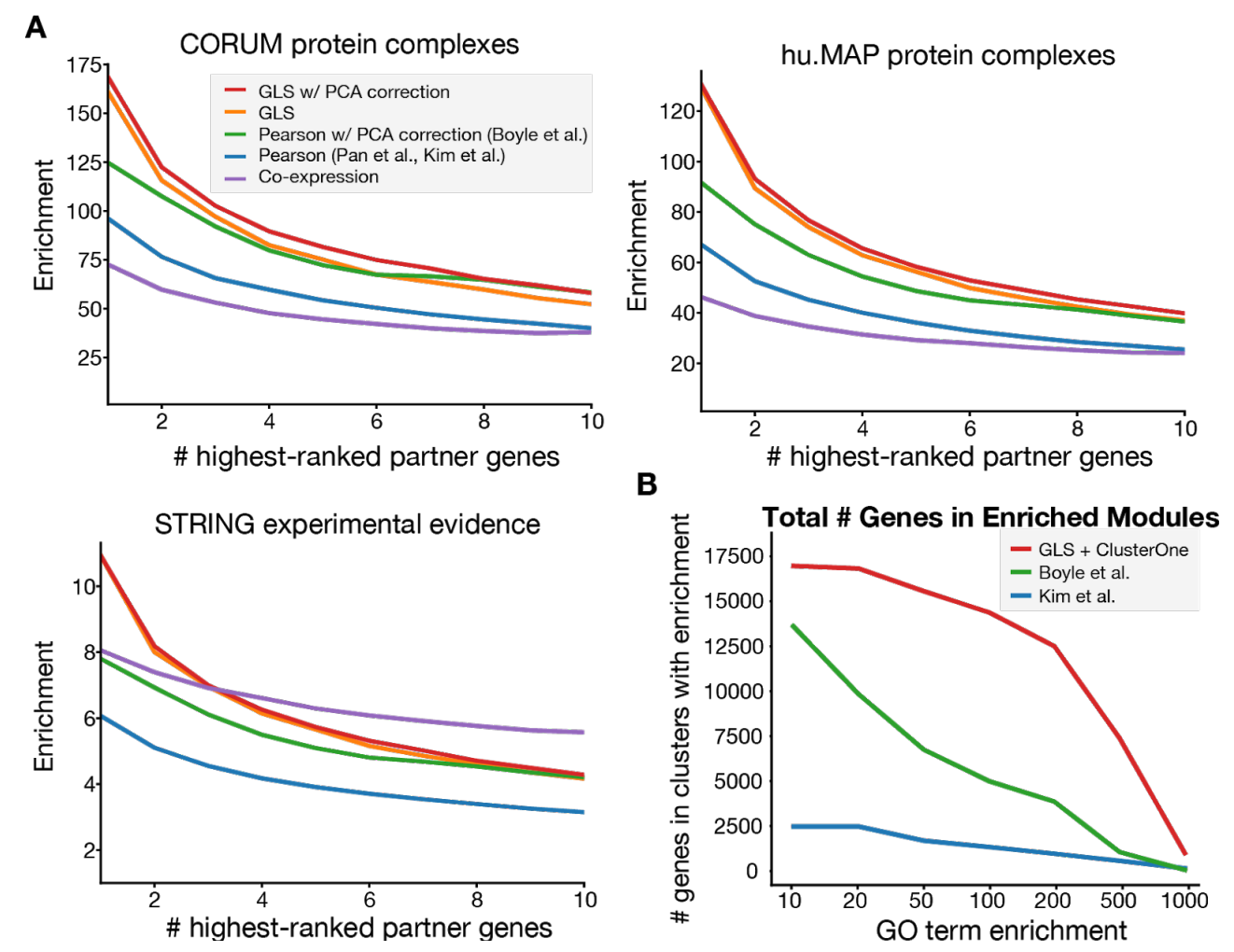
568 Figure 2: GLS improves recall of known functional interactions in co-essential gene pairs

569 and modules.

570 (A) Enrichment of interactions from GLS- and Pearson-based co-essentiality (with/without PCA-

571 based bias correction) using the DepMap dataset, as well as co-expression using the

572 COXPRESdb dataset, in CORUM, hu.MAP and STRING, considering the top 1-10 partners per

573 gene.

574 (B) Number of genes in clusters/modules at least $\mathrm{N}$-fold enriched for some $\mathrm{GO}$ term, excluding

575 the gene itself from the enrichment calculation, for various $\mathrm{N}$ from 10 to 1000.

576 See also Figures S2, S3, S6 and S7

577 
bioRxiv preprint doi: https://doi.org/10.1101/827071; this version posted November 3, 2019. The copyright holder for this preprint (which was not certified by peer review) is the author/funder. All rights reserved. No reuse allowed without permission.

\section{Figure 3}

\section{A Module \#1431: mTORC1 Regulation}

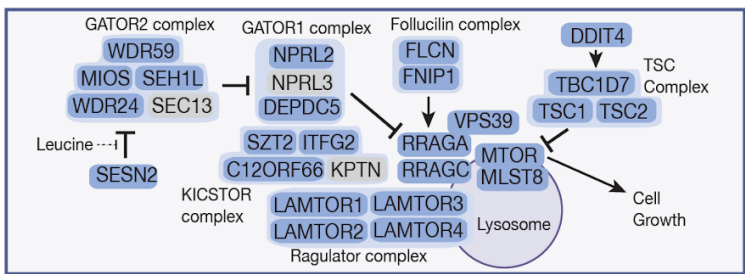

C Module \#520: Autophagy

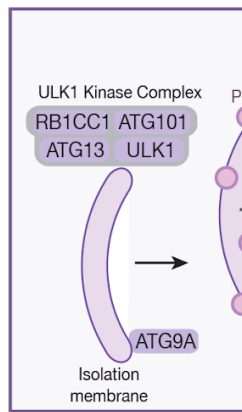

\section{E Module \#595: p53 Signaling}

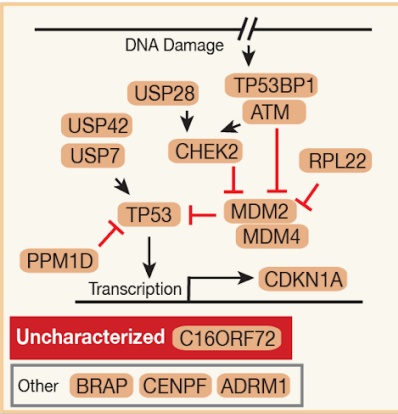

\section{Module \#1311: snRNA Transcription}

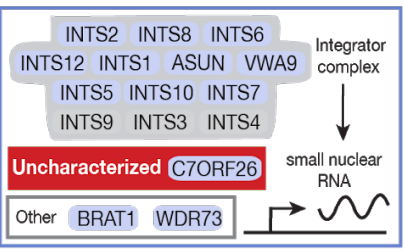

\section{B Module \#1193: mTORC2/PI3K/AKT signaling}

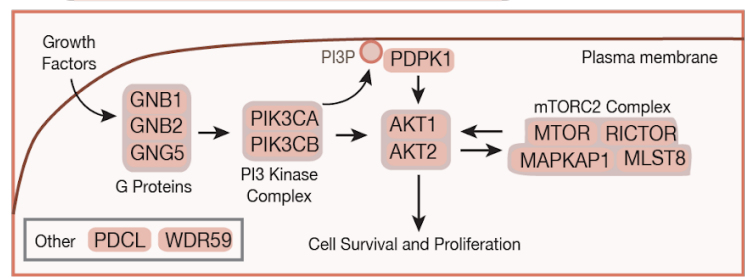

D Module \#307: Notch Signaling

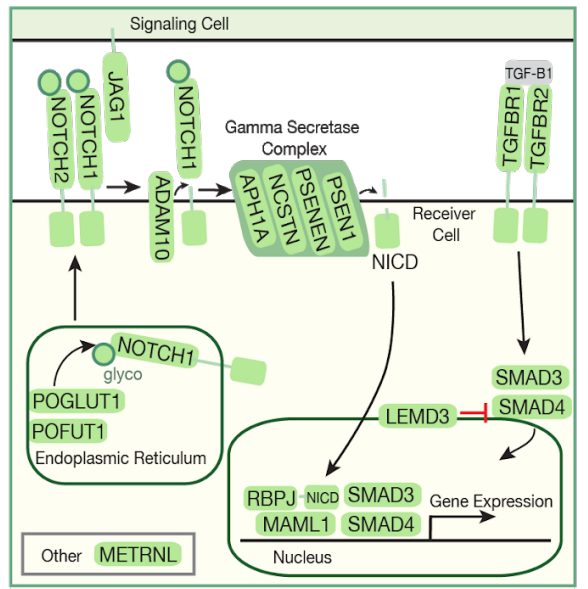

H Module \#473: Chromatin Remodeling

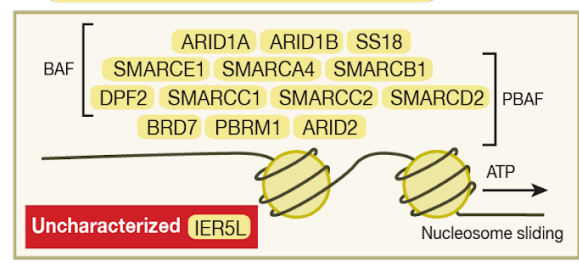

\section{J Module \#405: Cell Cycle}

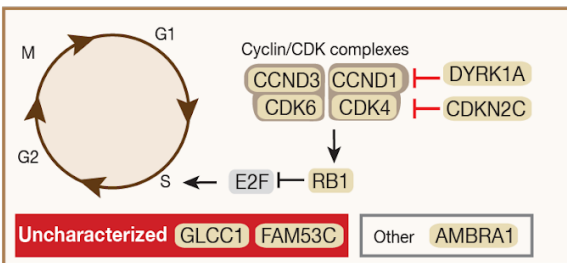

\section{K Module \#256: Mitochondrial respiration}

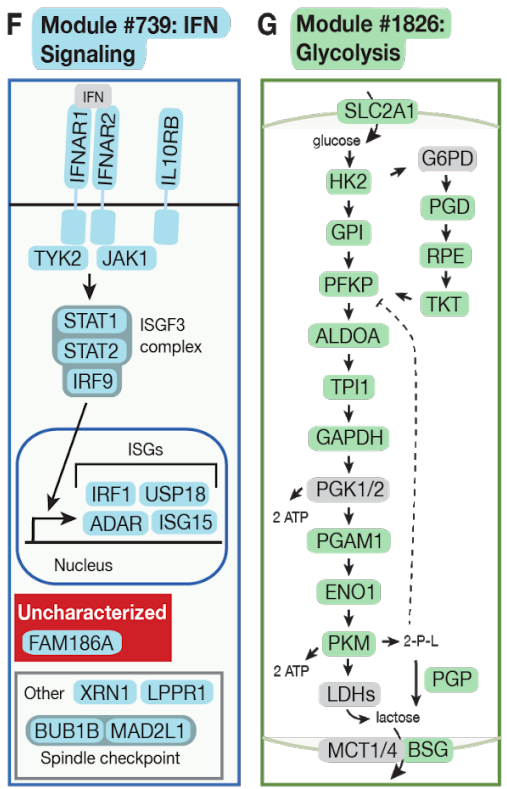

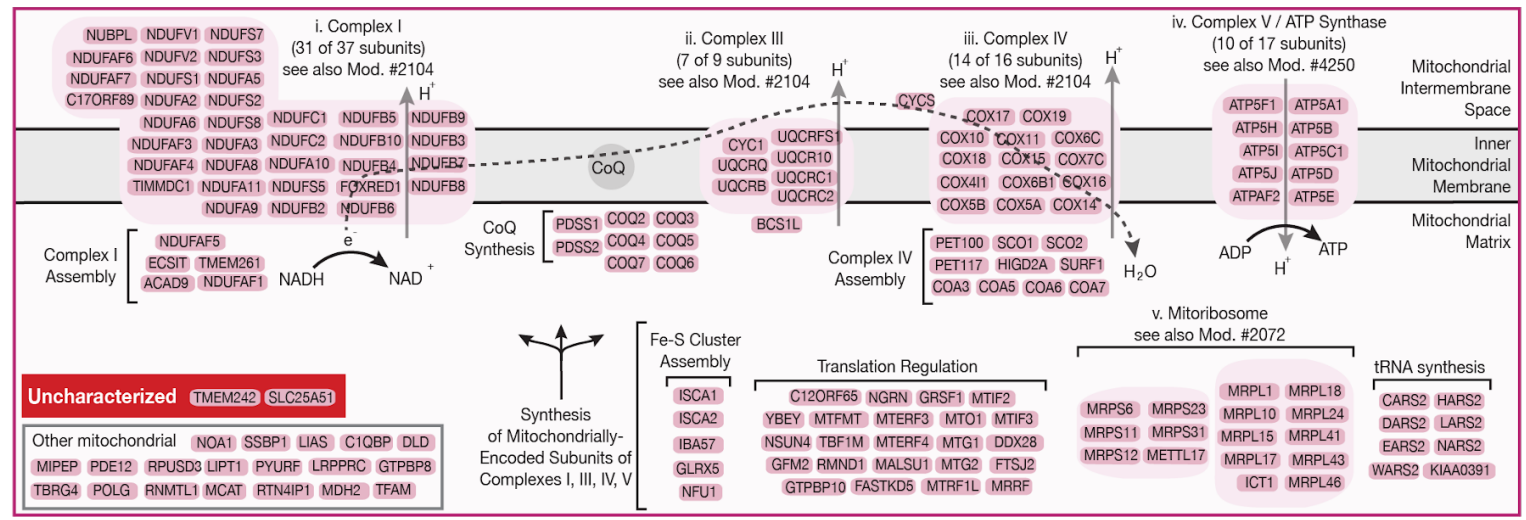


578 Figure 3: Co-essential modules recapitulate known pathways and nominate novel pathway

579 members.

580 (A-K) 10 examples of co-essential modules; all genes in each module are shown. Genes without

581 previous evidence of pathway involvement are indicated as either "uncharacterized" (Uniprot

582 annotation score <3) or "other". Red inhibitory arrows between gene pairs indicate both negative

583 regulation and negatively correlated essentiality profiles. In (A), (C), (G), (I), and (J), core pathway

584 members not included in the module are shown in gray. In (K), subunit counts for mitochondrial

585 respiration complexes were based on HGNC gene sets as of Oct 2019 (Povey et al., 2001).

586 Abbreviations: (B, C) PI3P, phosphatide-inositol-3-phosphate; (C) LC3s, Microtubule-associated

587 1A/1B-light chain (LC3) family members; (D) NICD, Notch intracellular domain; glyco, fucose and

588 glucose modifications transferred to NOTCH1 by POFUT1 and POGLUT1; TGF-B1, transforming

589 growth factor beta 1; (F) IFN, interferon; ISGs, interferon-stimulated genes; (G) 2-P-L, 2-phospho-

590 lactate (toxic byproduct of PKM) (Collard et al., 2016); (H) BAF, BRG- or HBRM-associated factors

591 complex; PBAF, polybromo BAF complex; (K) Mod., module; CoQ, coenzyme Q. 


\section{Figure 4}

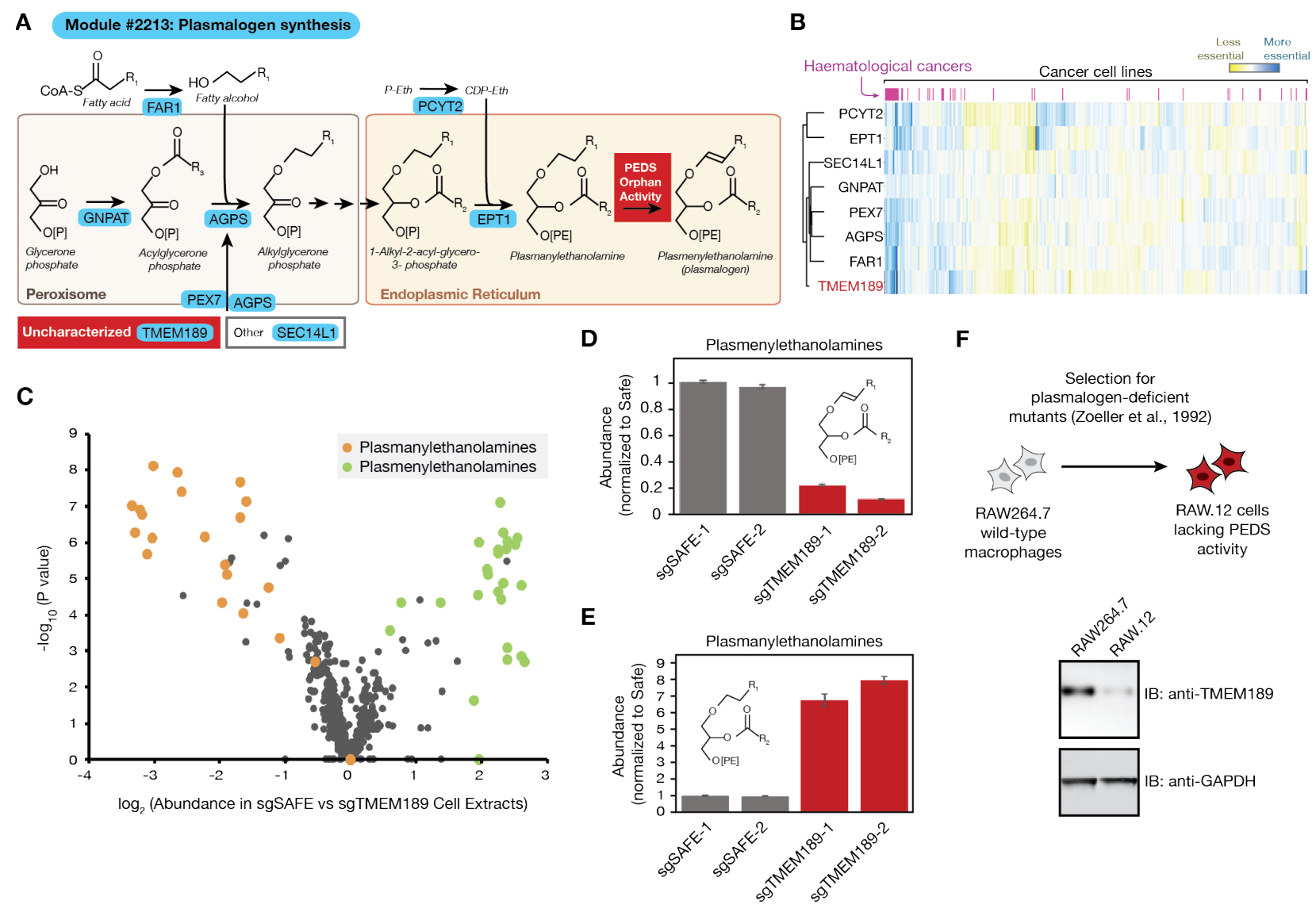



required for synthesis of plasmalogen lipids.

594 (A) Schematic of module \#2213 with manual annotations of gene function. Uncharacterized gene 595 selected for validation shown in red box. PEX7 is shown importing cytosolic AGPS across the 596 peroxisomal membrane into the peroxisome matrix (Braverman et al., 1997). 597 Plasmanylethanolamine desaturase (PEDS) orphan enzyme activity indicated in orange. P-eth, 598 phosphoethanolamine; CDP-Eth, cytidine diphosphate ethanolamine.

599 (B) Heatmap of bias-corrected essentiality scores of genes in module 2213 in 485 cancer cell 600 lines.

601 (C) Volcano plot of all lipid species detected in lipidomic experiment, with ratio of lipid abundance 602 in extracts derived from sgSAFE-1-expressing cells relative to sgTMEM189-1-expressing cells 603 plotted on x-axis.

604 (D) Total abundance (relative to Safe-targeting sgRNA control \#1) of 37 unambiguously identified 605 plasmenylethanolamine species in cell extracts prepared from HeLa cells transduced with 606 indicated sgRNAs. Error bars represent standard deviation ( $n=4$ technical replicates).

607 (E) Total abundance (relative to Safe-targeting sgRNA control \#1) of 30 unambiguously identified 608 plasmanylethanolamine species in cell extracts prepared from HeLa cells transduced with 609 indicated sgRNAs. Error bars represent standard deviation ( $n=4$ technical replicates).

610 (F) Top, schematic of generation of RAW.12 derivative of RAW264.7 macrophage-like line with 611 confirmed deficiency in plasmanylethanolamine desaturase (PEDS) activity, as reported in Zoeller 612 et al., 1992. Bottom, immunoblotting (IB) with anti-TMEM189 antibodies in RAW264.7 parental 613 line and RAW.12 PEDS-deficient line.

614 See also Figure S4. 


\section{Figure 5}

A Module \#2067: Clathrin adaptor activity

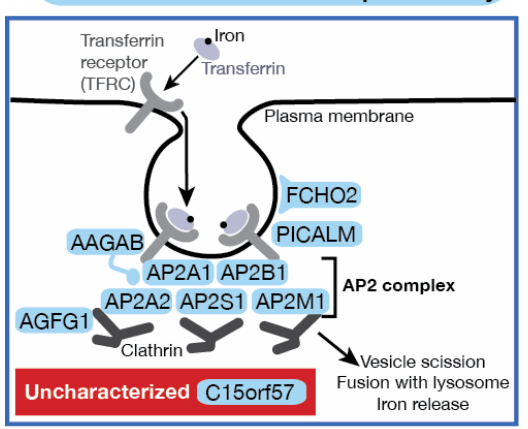

C

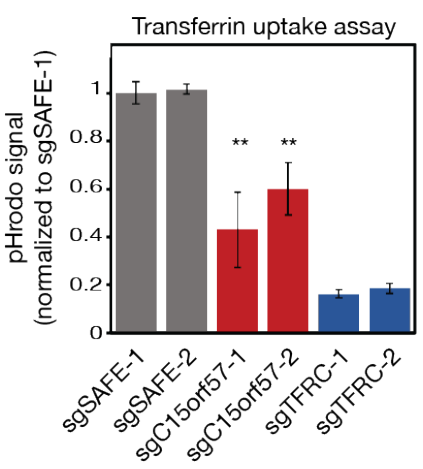

E

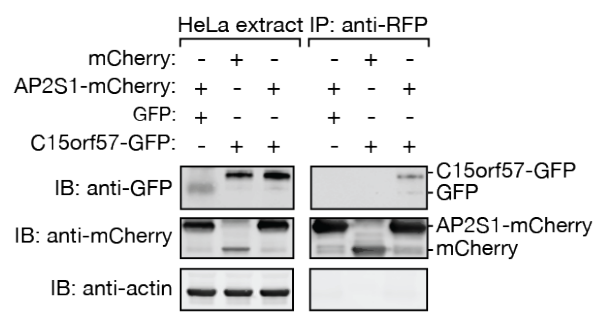

B

D
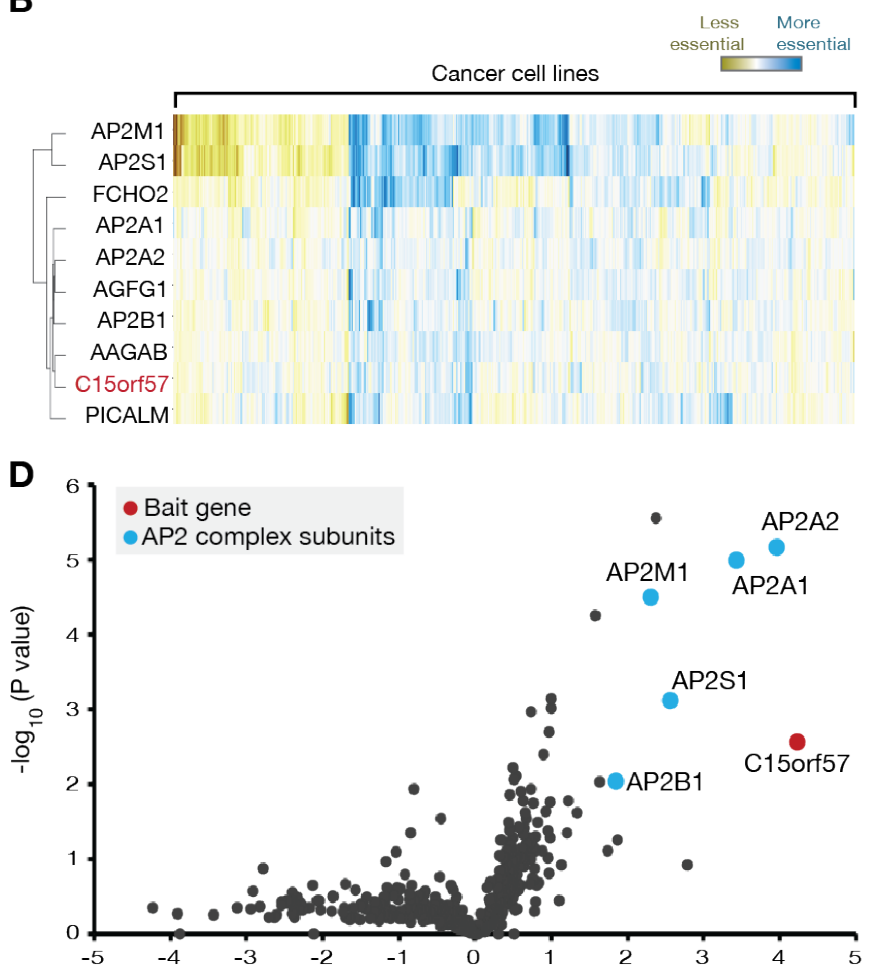

$\log _{2}$ (Abundance in C15orf57-GFP IPs vs control IPs)

F

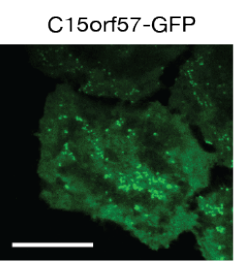

AP2S1-mCherry

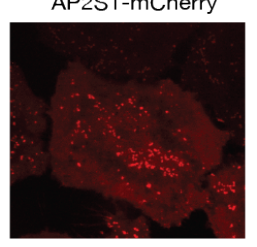

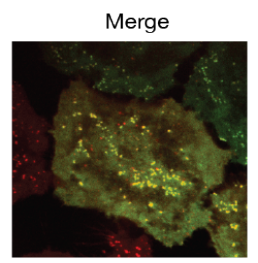


615 Figure 5: C15orf57 is required for efficient clathrin-mediated endocytosis of transferrin.

616 (A) Schematic of module \#2067. Uncharacterized gene selected for validation shown in red. AP2, 617 adaptor protein 2.

618 (B) Heatmap of bias-corrected essentiality scores of genes in module \#2067 in 485 cancer cell 619 lines.

620 (C) Transferrin-pHrodo uptake assay for clathrin-mediated endocytosis (24h timepoint). Error bars

621 represent standard deviation $\left(n=3\right.$ technical replicates, two-tailed Student's t-test, $\left.{ }^{* *} p<.01\right)$. Data

622 shown are representative of three independent experiments.

623 (D) Volcano plot of mass spectrometric (TMT) analysis of C15orf57-GFP immunoprecipitates 624 (IPs).

625 (E) Extracts prepared from indicated HeLa cell extracts were subjected to immunoprecipitation 626 with anti-RFP magnetic resin. Extracts and IP samples were resolved by SDS-PAGE and followed 627 by immunoblotting with indicated antibodies.

628 (F) Microscopy of HeLa cells transduced with C15orf57-GFP and AP2S1-mCherry constructs. 629 Scale bar, $20 \mu \mathrm{m}$. 


\section{Figure 6}

A

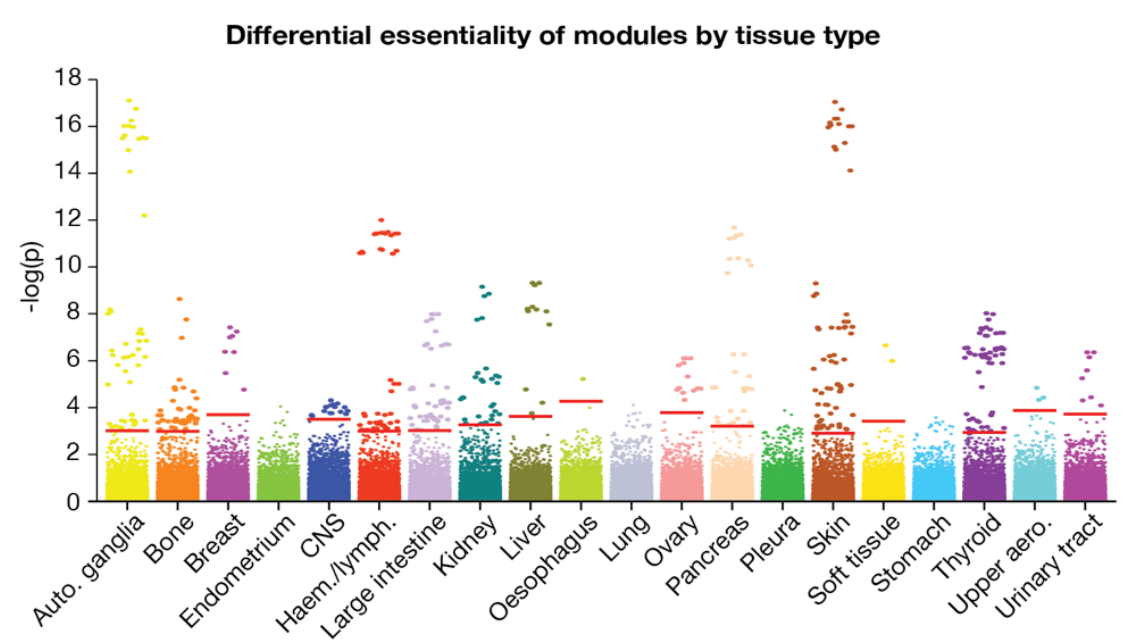

B

Gene essentiality in breast cancer cell lines

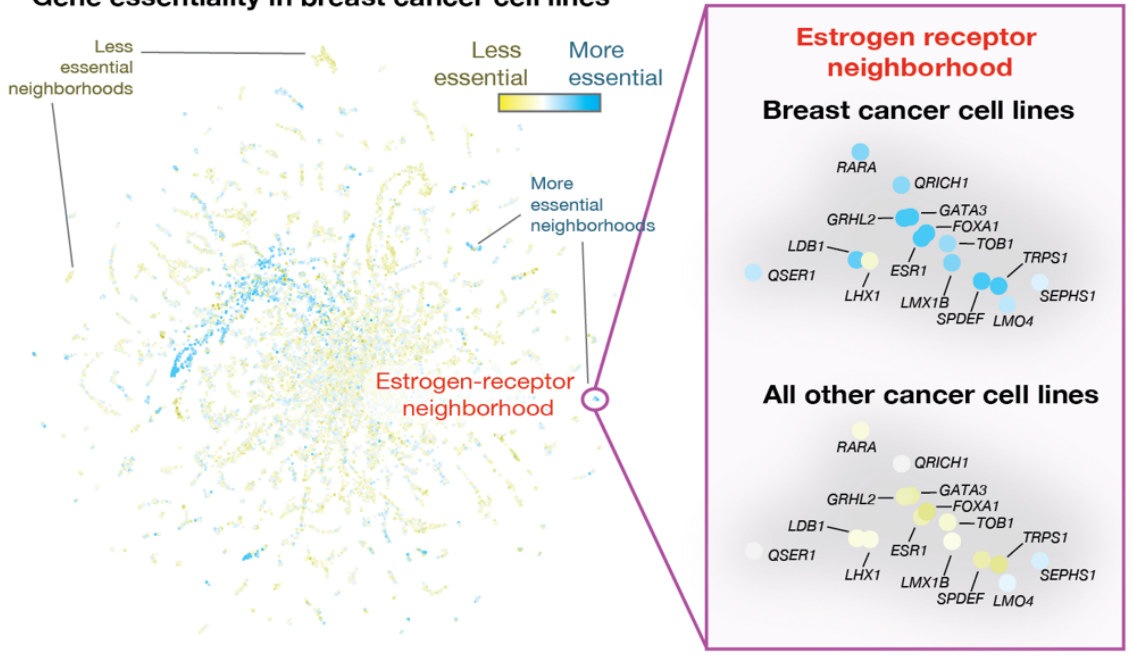

C

Gene essentiality in skin cancer cell lines

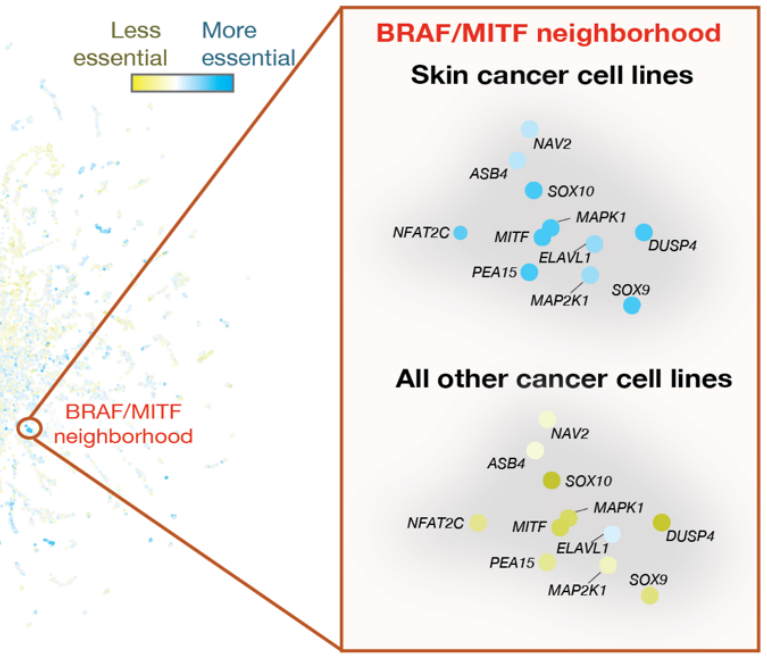


630 Figure 6: Identification of cancer type-specific module dependencies.

631 (A) Differential essentiality of co-essential modules in cell lines derived from 20 tissue types. -

$632 \log _{10}(p$-values $)$ for each module are plotted for each tissue (see Methods). Red bars indicate FDR

633 thresholds for each tissue type. Auto., autonomic; CNS, central nervous system; Haem.,

634 haematological; lymph., lymphoma; aero., aerodigestive.

635 (B) Average bias-corrected gene essentiality in breast cancer cell lines plotted on two-

636 dimensional co-essentiality network, with gene neighborhood containing ESR1 highlighted on the 637 right.

638 (C) Average bias-corrected gene essentiality in skin cancer cell lines plotted on two-dimensional 639 co-essentiality network, with gene neighborhood containing BRAF and MITF highlighted on the 640 right.

641 See also Figure S5, Video S1. 


\section{Figure S1: Co-essentiality profiling and the limitations of Pearson correlation}

645 (A) The concept of co-essentiality: (left) a pair of functionally related genes are both essential in 646 some cell lines and both non-essential in other lines. Essentiality can be quantified from CRISPR 647 screens as the logarithm of the growth effect of the gene's knockout (intuitively, the number of 648 times fewer cells with the knockout doubled during the screen, compared to control cells). (Right) 649 a pair of unrelated genes have uncorrelated essentiality across cell lines.

650 (B) Simulation of how biological relatedness between cell lines inflates Pearson correlation $p$ 651 values. Duplicating each point 10 times with slight noise (analogous to duplicating each screen in 65210 related lines) makes the previously non-significant $(p=0.6)$ blue correlation highly significant $653(p=0.007)$ and the significant red correlation $\left(p=7 \times 10^{-5}\right)$ substantially more so $\left(p=2 \times 10^{-103}\right)$, 654 despite similar correlation magnitudes.

Figure S2: Number of co-essential partners per gene by average gene essentiality

657 Number of co-essential partners at $1 \%$ and $10 \%$ FDR as a function of a gene's average essentiality (pre-bias-correction CERES score) across lines.

\section{Figure S3: Benchmarking of cluster density $d$}

661 F1 score (harmonic mean of precision and recall) for various values of the module density 662 parameter $d$ on CORUM, hu.MAP and STRING. F1 scores represent the performance of a binary 663 network based on the modules (i.e. "are genes A and B in the same module?") at predicting a 664 binary network based on the benchmark dataset (i.e. "are genes A and B partners in the 665 benchmark dataset?"). 

in sphingolipid biosynthesis

669 (A) Abundances (relative to Safe-targeting sgRNA control \#1) of very long chain sphingomyelin 670 species (with acyl chain length indicated on x-axis) in cell extracts prepared from HeLa cells 671 transduced with indicated sgRNAs. sgSafe data and sgTMEM189 data are from same data set 672 represented in Figure 4C.

673 (B) Volcano plot of mass spectrometric (TMT) analysis of TMEM189-GFP immunoprecipitates.

674 Data are from same mass spectrometry analysis as data shown in Figure 4D.

\section{Figure S5: A web tool for interactive exploration of the co-essential network}

677 Example use case for the interactive web tool (coessentiality.net). A gene, KRAS, was selected 678 using the dropdown menu at top left and is marked with a red arrow in the scatterplot below. Genes selected for analysis - KRAS and its gene neighborhood - are designated with red points in the main panel (left). The heatmap panel (top right) shows that KRAS-mutant lines (selected for display using the search bar above the heat map and indicated as black marks in the "Mutation" bar above the heatmap) are enriched in a cluster (far right) that is marked by increased essentiality of KRAS. The pathway enrichment panel (bottom right) shows strong enrichments for Ras signaling and related pathways. The points in the main panel have also been selected in the tissue search bar (top middle) to be colored according to the average essentialities of each gene in

686 kidney-derived cell lines. Gene sets can also be either saved or uploaded as csv files using the 687 respective buttons in the top center (under "Gene set download/upload"). Some web colors and 688 font sizes were optimized for display in this figure. 
Figure S6: Benchmarking of syntenic versus non-syntenic modules

694 Enrichment of syntenic (both genes on same chromosome) and non-syntenic co-essential pairs

695 for annotated interactions CORUM, hu.MAP and STRING databases, using the same 696 benchmarking strategy as in Figure 2.

Figure S7: Number of genes assigned putative functions by various co-essentiality module detection methods, after excluding syntenic modules.

700 Number of genes in non-syntenic clusters/modules at least N-fold enriched for some GO term, 701 excluding the gene itself from the enrichment calculation, for various $\mathrm{N}$ from 10 to 1000.

702

Table S1: Spreadsheet of significant co-essential interactions at $10 \%$ per-gene FDR.

704 List of all co-essential gene pairs identified in this study, with the number of Pubmed citations (as 705 of Oct 2019) and chromosome location for each gene, and the direction of the gene correlation 706 (positive $(+)$ or negative $(-))$.

707

Table S2: Co-essential and co-expressed partners of TP53, KRAS and BRCA1.

709 Significant GLS co-essential versus top co-expressed partners of TP53, KRAS and BRCA1.

710 Genes in bold have strong evidence of being part of the same pathway.

712 Table S3: Spreadsheet of co-essential modules.

713 List of all 5,228 co-essential modules and their constituent genes, with top 3 most-enriched gene 714 ontology terms and their associated enrichments and $p$-values, the value of $d$ used to define the 715 module, and a link to the heatmap of batch-corrected essentiality data across 485 cell lines. 
719 Table S4: Uncharacterized gene functional predictions.

720 List of uncharacterized genes that are present in co-essential modules $>100$-fold enriched for a

721 gene ontology term, the Uniprot annotation score and number of Pubmed citations for each gene

722 (as of Oct 2019), and the set of genes in each cluster that is and is not annotated with the most-

723 enriched gene ontology term.

724

725 Table S5: Lipidomics data.

726 Lipid species concentrations for indicated lipids measured using Lipidyzer platform in indicated

727 cell lines. QC1, QC2, and QC3 indicate quality controls (see Methods).

728

729 Table S6: Mass spectrometry data for proteomic analysis of C15orf57 and TMEM189

730 interactomes.

731 Proteomic data, including complete list of proteins and enrichment $p$-values, for C15orf57 and

732 TMEM189 interactome analyses in Figures 4 and 5.

733

734 Table S7: Cancer type-specific module dependencies.

735 List of 444 differentially essential modules across 16 tissue types, ranked by $p$-value.

736

737 Video S1: Example use cases of co-essential browser.

738 Guide to use of co-essential browser showing how to navigate web tool in the context of multiple 739 use cases, including gene lookup, gene set selection, and gene list upload. 


\section{METHODS}

\section{Code availability}

742 Code to generate co-essential gene pairs, co-essential modules, modules with cancer type-

743 specific dependencies, and the two-dimensional layout will be made available at

\section{4 https://github.com/kundajelab/coessentiality.}

746 Dataset

747 The dataset used to determine co-essential interactions consists of the 485 genome-wide

748 CRISPR screens from the Achilles project 18Q3 release (Tsherniak et al., 2017). Specifically,

$749 \quad 17,634$ genes were screened in 485 cell lines from 27 distinct lineages using the Avana CRISPR

750 library (Doench et al., 2016), and gene-level effects were quantified using the CERES algorithm

751 to account for variability in guide effectiveness and copy number across lines (Meyers et al.,

752 2017), resulting in a 17,634 x 485 matrix of normalized gene-level effects. Intuitively, gene-level

753 effects represent the number of times fewer cells with the knockout doubled during the screen,

754 compared to control cells. This dataset is publicly available at

755 https://ndownloader.figshare.com/files/12704099, or at https://depmap.org/portal/download/all/

756 under release "DepMap Public 18Q3" and file "gene_effect.csv".

\section{Bias correction}

759 Bias correction was applied as described in Boyle et al., 2018. Specifically, the first 4 principal

760 components of the gene-by-cell-line essentiality matrix across all olfactory receptor genes,

761 defined here as those with the "olfactory receptor activity" gene ontology (GO) term (Ashburner

762 et al., 2000; The Gene Ontology Consortium, 2017), were subtracted from the original CERES

763 score matrix, resulting in a new bias-corrected matrix. To avoid multicollinearity and allow

764 inversion of the covariance matrix for generalized least squares (see below), subtraction of the 
765 first 4 principal components was followed by removal of 4 cell lines (arbitrarily chosen to be the 766 last 4), resulting in a 17,634-by-481 matrix of bias-corrected CERES scores.

\section{Quantifying co-essential gene pairs}

769 The co-essentiality between each pair of genes was quantified using generalized least squares 770 (Aitkin, 1935). In a departure from previous approaches to co-essentiality profiling, GLS

771 automatically and flexibly accounts for the non-independence of cell lines by incorporating

772 information about the covariation between every pair of screens. When all screens are

773 independent and have the same variance in effect sizes across genes, the GLS effect size

774 becomes exactly equivalent to the Pearson correlation coefficient. GLS is closely related to the

775 linear mixed models (LMMs) used for population structure correction in genome-wide association

776 studies (Yu et al., 2006), an analogous problem to ours.

778 Specifically, GLS estimates the vector of parameters $\boldsymbol{\beta}$ of the linear regression model $\mathbf{Y}=\mathbf{X} \boldsymbol{\beta}+\boldsymbol{\varepsilon}$, 779 where $\mathrm{Y}$ is a vector of observations, $\mathbf{X}$ is a matrix of features corresponding to those observations, 780 and $\varepsilon$ are the errors or residuals, under the assumption that the mean of the errors is 0 and their 781 variance is $\Sigma$, where $\Sigma$ is a covariance matrix specified by the practitioner. The only difference from

782 ordinary least squares (OLS) is the value of $\Sigma$; OLS assumes that it is the identity matrix, while

783 GLS allows it to be any user-specified value. Here, we set $\Sigma$ to be the covariance matrix of the

784 data itself, i.e. $V_{i, j}$ is the covariance of cell lines $i$ and $j$ across all genes in the CRISPR screen.

785

786 In practice, GLS is solved by a) inverting $\Sigma$, in our implementation

787 (statsmodels.regression.linear_model.GLS from the statsmodels Python package) by using the

788 Moore-Penrose pseudoinverse instead of the true inverse as a computational optimization, b)

789 taking the Cholesky decomposition of this inverse covariance matrix chol $\left.\left(\Sigma^{-1}\right), \mathrm{c}\right)$ transforming both 
$\mathbf{Y}$ and $\mathbf{X}$ by $\operatorname{chol}\left(\Sigma^{-1}\right)$ to obtain the transformed observations $\mathbf{Y}^{\prime}=\operatorname{chol}\left(\Sigma^{-1}\right) Y$ and transformed features $\mathbf{X}^{\prime}=\operatorname{chol}\left(\Sigma^{-1}\right) \mathbf{X}$, and d) running OLS on $\mathbf{Y}^{\prime}$ and $\mathbf{X}^{\prime}$. (When $\Sigma$ is the identity matrix, $\operatorname{chol}\left(\Sigma^{-1}\right)$ is as well, so $\mathbf{Y}^{\prime}=\mathbf{Y}$ and $\mathbf{X}^{\prime}=\mathbf{X}$ and GLS reduces to $\mathrm{OLS}$.)

GLS was run separately on each gene pair, resulting in a 17,634-by-17,634 matrix of GLS $p$ values. Specifically, the endog argument of statsmodels.regression.linear_model.GLS (the output) was set to the length-481 vector of bias-corrected CERES scores for one of the two genes,

797 the exog argument (input) set to a 481-by-2 matrix where the first column is the other gene's bias-

798 corrected CERES scores and the second column is a constant vector of all ones (i.e. the 799 intercept), and the sigma argument set to the 481-by-481 covariance matrix of the bias-corrected

800 CERES scores. Given these three pieces of data, the GLS outputs a $p$-value indicating the 801 statistical significance of the degree of co-essentiality between the pair of genes. Note that while 802 the GLS $p$-value is consistent regardless of which of the two genes is chosen as endog and which

803 as exog, the GLS effect size is not consistent with respect to this choice, and as a result is not

804 reported. For benchmarking, GLS was also run on the non-bias-corrected data using the exact same procedure, but using the full 485 cell lines.

807 As a computational optimization, the rate-limiting step of the GLS calculation (inverting the 808 covariance matrix and then taking the Cholesky decomposition) was cached and reused for each 809 pair of genes, since all gene pairs use the same covariance matrix. With this optimization, the 810 amortized time complexity of GLS is equivalent to Pearson correlation. The same GLS 811 implementation was used to calculate the Pearson correlation (with and without bias correction) 812 between each pair of genes, by setting the covariance matrix to the identity matrix. 


\section{Pearson correlation simulations}

816 For the simulations in Figures S1A and B, the $x$ coordinates of the 8 red data points were sampled

817 to be uniformly distributed between -4 and 0 . The y coordinates were then sampled from $0.9 x+$

818 Normal $\left(0,\left(1-0.9^{2}\right)^{1 / 2}\right)$ to have a Pearson correlation of approximately 0.9 . To be visually pleasing,

819 points were repeatedly re-simulated until two constraints were satisfied: the most extreme $\mathrm{x}$ and

820 y coordinates had to be between 0.15 and 0.4 from the edge of the interval $[-4,0]$, and the

821 minimum $x$ and $y$ differences between each pair of points had to be at least 0.2 .

822

823 A second set of blue points were added alongside the red points. The blue points and red points

824 share the same $x$ coordinates, but the blue points' y coordinates were sampled to be uniformly

825 distributed between -1 and 0 to avoid having any significant correlation with the $x$ coordinates. To

826 enforce this lack of correlation, the y coordinates were repeatedly sampled until both the Pearson

827 and Spearman correlation $p$-values were greater than 0.5 .

829 In the right half of Figure S1B, the same red and blue points were plotted, in addition to 20

830 duplicates of each of these points, shifted by a small amount of noise sampled from $\operatorname{Normal}(0$,

$8310.1)$

\section{Benchmarking on CORUM, hu.MAP and STRING}

834 For the benchmarking in Figure 3, we compared five methods: co-essentiality with GLS or

835 Pearson and with or without bias correction, and co-expression with COXPRESdb. We used the

836 same versions of COXPRESdb benchmarked in Pan et al., downloaded from the supplemental

837 data to that paper at https://ndownloader.figshare.com/files/10975364 and remapped from Entrez

838 IDs to gene names using the mapping at https://ndownloader.figshare.com/files/9120082. When

839 benchmarking, we considered only the $N=15,552$ genes present in both the Avana library and

840 COXPRESdb. 
842 For STRING, we used all the gene pairs in version 10.5 restricted to Homo sapiens

\section{3 (https://stringdb-}

844 static.org/download/protein.links.detailed.v10.5/9606.protein.links.detailed.v10.5.txt.gz).

845 avoid circularity, we removed gene pairs supported only by co-expression, i.e. for which the only

846 non-zero score was for co-expression.

848 Following the strategy of Pan et al., we compared methods by considering their rankings on a

849 per-gene basis. Specifically, we considered only the top $\mathrm{N}$ partners for each gene for $\mathrm{N}$ from 1 to

85010 , and looked at how enrichment varied as a function of $\mathrm{N}$. We used the same versions of

851 CORUM and hu.MAP benchmarked in Pan et al.

853 Enrichments were calculated as the percent of the top $\mathrm{N}$ gene pairs in the pathway or complex 854 database, divided by the percent of gene pairs found in the database. For instance, to calculate 855 the enrichment of COXPRESdb in CORUM for $N=2$, we found the top 2 co-expressed partners 856 per gene according to COXPRESdb ( $N=2$ * 15,552 gene pairs), computed the percent of these 857 pairs that were part of the same CORUM complex, and divided by the percent of the 15,552 * 85815,552 gene pairs that were part of the same CORUM complex.

860 Note that Boyle et al. perform an additional transformation of $p$-values after PCA correction based 861 on the empirical null distribution of $p$-values for olfactory genes, but since this transformation is 862 monotonic it does not affect the rankings of partner genes used in our benchmarking.

\section{Co-essential modules}

865 Co-essential modules were ascertained with the ClusterONE algorithm (Nepusz et al., 2012). 866 Briefly, ClusterONE generates modules by greedily adding nodes (genes) starting from a 
randomly selected seed node, so long as the sum of the edge weights within the module is

868 sufficiently high relative to the sum of the boundary edge weights between genes in the module

869 and their neighbors. It then merges sufficiently overlapping modules as a post-processing step,

870 while allowing genes to be members of multiple modules (protein complexes or pathways).

872 ClusterONE was run on the 17,634-by-17,634 matrix of GLS p-values after row-wise false

873 discovery rate correction, with edge weights set to one minus the false discovery rate q-value

874 (Storey and Tibshirani, 2003). Default settings were used for ClusterONE, except for changing

875 the module density parameter -d (also known as --min-density) from its default of 0.3 , as

876 discussed in the main text. For the list of modules in Table S3, all modules generated with values

877 of $\mathrm{d}$ set to $0.2,0.5$, and 0.9 were merged into a single list. 11 modules that were identical at

878 different values of $d$ were retained in this list but were excluded from the reported count of total

879 modules.

881 We noted that the resulting list of co-essential modules contained many modules that are highly 882 enriched for genes that localize close to one another in the genome. In several cases, these 883 modules correspond to clusters of functionally related genes that are known to colocalize in the 884 genome, such as histone- and protocadherin-encoding genes, though in the majority of cases it 885 remains unclear whether the presence of colocalized genes in a module reflects their shared 886 function in a biological pathway or if it relates to vulnerabilities of CRISPR screening to copy887 number artifacts that are difficult to account for perfectly (Meyers et al., 2017). Supporting the idea 888 that co-essentiality for colocalized genes may represent a mix of true- and false-positive signals, 889 we find substantial enrichment of syntenic gene pairs (both genes on the same chromosome) in 890 CORUM, hu.MAP and STRING, but less enrichment than for non-syntenic gene pairs (Figure 891 S6). We note that even after excluding syntenic modules (i.e. those that contain genes which are 892 all located on the same chromosome), our set of co-essential modules still assigns putative 
893 functions to approximately 10,000 genes using the metric described earlier in relation to Figure

894 2B (i.e.the number of genes included in a module that is at least 100 -fold enriched for some GO

895 term), approximately twice as many as the next-best method (Figure S7). To enable full utilization

896 of the dataset as well as easy discernment of syntenic and non-syntenic gene pairs and modules,

897 we report all co-essential gene pairs and modules in Tables S1 (co-essential pairs), S3 (co-

898 essential modules) and S4 (uncharacterized gene predictions) and annotate each as syntenic or

899 non-syntenic.

900

901 Identification of cancer type-specific pathway dependencies

902 Cancer type-specific pathway dependency $p$-values for each module and cancer type (Table S7)

903 were obtained by 1) computing $p$-values for each gene and cancer type, then 2) aggregating $p$ -

904 values across genes in each module. In step 1), GLS was run separately for each gene with the

905 same covariance matrix and output/endog argument (bias-corrected essentiality for a particular

906 gene) as before (see "Quantifying co-essential gene pairs"). However, unlike before, the exog

907 argument (input) was set to a 481-by-21 matrix of binary indicator variables for the 20 cancer

908 types listed in Figure 6A ( 1 if a cell line is from that cancer type, 0 otherwise) plus an all-ones

909 intercept column. The two other cancer types with CRISPR screen data from DepMap, cervical

910 and biliary, were excluded due to only having a single cell line each. This multiple regression

911 yielded 20 -values for the gene, one per cancer type. We note that this approach is equivalent

912 to an ANOVA, except using GLS instead of OLS.

914 In Step 2), p-value aggregation was performed separately for each module and cancer type using

915 the Cauchy Combination Test/Aggregated Cauchy Association Test (Liu and Xie, 2019; Liu et al.,

916 2019) with equal weights on all genes. In Python, this step can be expressed straight-forwardly

917 as "module_p = cauchy.sf(np.tan((0.5 - gene_ps) * np.pi).mean())", where gene_ps is a (number

918 of module genes)-length vector of gene $p$-values for a particular cancer type, and module $p$ is 
919 the combined $p$-value for the module. Crucially, given that our gene-level $p$-values are highly

920 correlated among genes in a module, the test is able to accommodate $p$-values from correlated

921 tests (unlike the more commonly used Fisher's combined $p$ test, which uses a chi-squared instead

922 of a Cauchy distribution to perform $p$-value aggregation), and we verified that the combined $p$ -

923 values were not inflated (median $p$-value $=0.56)$.

924

\section{Global structure of the co-essential network}

926 The two-dimensional interaction map visualization was constructed to have two properties: (a)

927 genes in many of the same ClusterONE modules are close together; (b) gene pairs with high GLS

928 co-essentiality are close together. This was done by forming a graph $\mathrm{G}_{\mathrm{co}}$ from the ClusterONE

929 modules (as above) and another $\mathrm{G}_{\mathrm{GLS}}$ from the co-essentiality data, mixing the two with proportion

$930 \quad \alpha$ to form the mixed graph:

931

932

$\mathrm{G}=\alpha \mathrm{G}_{\mathrm{CO}}+(1-\alpha) \mathrm{G}_{\mathrm{GLS}}$

933

934 (We set $\alpha=0.99$ to rely on the relatively specific and dense ClusterONE modules where possible,

935 while falling back on pairwise GLS analysis to link genes not in any module to the rest of the 936 network.)

938 The graph $\mathrm{G}_{\mathrm{GLS}}$ was constructed by computing, for each pair of genes, -log(p) given by GLS 939 between the two genes. This was denoised and compressed by keeping each gene's edges to its

94010 nearest neighbors and zeroing the other edges, resulting in each gene having a minimum of

94110 neighbors in the graph. (We found our analyses fairly stable to varying the number of nearest

942 neighbors between 4 and 100.) The graph $\mathrm{G}_{\mathrm{co}}$ was constructed using the same procedure, but

943 with each pairwise similarity computed using the Jaccard similarity between the sets of 
944 ClusterONE modules the respective genes belonged to (for sets $A$ and $B$, this is $J(A, B)=$ $945|A \cap B| /|A \cup B|)$.

946

947 To visualize the network G efficiently on a global scale, we relied on the framework of diffusion 948 maps (Coifman and Lafon, 2006), which basically decompose the variation in essentiality profiles 949 over the network into short- and long-range pathway components, resulting in an embedded

950 space for genes in the network. The genes' positions here are relatively accurate for genes in 951 well-separated pathways, and less so for finer distinctions - this embedded space (the "diffusion 952 map") is a smoothed version of the network, with each gene being represented in low dimension $953 d=40$. The embedded space was constructed from $G$ as follows.

955 G was first normalized to remove the disproportionate influence of high-degree "hub" genes in the 956 layout, resulting in a matrix $G_{2}$. With this gene-wise degree expanded as a matrix $D_{G}=\operatorname{diag}\left(\sum_{j} G_{i j}\right)$, 957 the normalization operation is:

958

$\mathrm{G}_{2}=\mathrm{D}_{\mathrm{G}}^{-1} \mathrm{GD}_{\mathrm{G}}^{-1}$

960

961 This density normalization further corrects for biased sampling of the network by the data 962 (Coifman and Lafon, 2006; Haghverdi et al., 2015), as analyses on $\mathrm{G}_{2}$ consider the gene network 963 corrected for the variable density of characterized genes.

964

965 The diffusion map embeds $\mathrm{G}_{2}$, and takes the properties of random walks on it to reveal multi966 scale pathway structure. The transition probabilities of such a random walk on $\mathrm{G}_{2}$ are the row967 sum-normalized $T=D_{2}^{-1} G_{2}$, where $D_{2}=\operatorname{diag}\left(\sum_{j}\left[G_{2}\right]_{i j}\right)$. 
969 This transition matrix T describes the evolution of any random walk, and its right eigenvectors

$970 \mathrm{e}_{1}, \ldots, \mathrm{e}_{\mathrm{n}}$ give a diffusion map embedding when appropriately scaled. The embedding requires a

971 parameter $\mathrm{t}$, which controls the overall scale of the pathways modeled by the embedding. If the

972 corresponding eigenvalues are $\lambda_{1} \geq \lambda_{2} \geq \ldots$, then for any $t>0$, the embedded coordinates of the

973 genes $\left[\Phi_{\mathrm{t}}\right]_{1},\left[\Phi_{\mathrm{t}}\right]_{2}, \ldots,\left[\Phi_{\mathrm{t}}\right]_{40}$ are:

974

$975\left[\Phi_{\mathrm{t}}\right]_{\mathrm{i}}=\lambda_{\mathrm{i}}^{\mathrm{t}} \mathrm{e}_{\mathrm{i}}$

976

977 A crucial choice is that of the scale parameter $t$. As the current co-essentiality data are some-

978 what noisy for inferring fine-grained gene-gene relationships, we found it necessary to smooth

979 them by increasing the value of $t$ in constructing the embedding. We increased to the minimum

980 such that $d=40$ dimensions captured $90 \%$ of the variance in the embedded space $\Phi_{\mathrm{t}}$, and

981 computed the resulting diffusion map $\Phi$. This simultaneous optimization of $t$ and $\Phi_{\mathrm{t}}$ made the

982 procedure adapt to and preserve large-scale global structure in a fully data-driven way, without

983 substantive parameter tuning and using only a few matrix multiplications and one SVD.

985 We applied UMAP (Mclnnes et al., 2018) to this diffusion map embedding as in scanpy for the

986 final global layout. Our diffusion maps implementation is in Python using the numpy and scipy

987 packages, and includes other choices of normalization as well. The entire process ran in less than

9884 minutes on the GLS- and ClusterONE-derived matrices on an Intel i7 Core CPU.

989

990 Browser heatmap

991 The 481-cell-line bias-corrected CERES essentiality scores are plotted alongside the global co992 essentiality network in the browser (Figure S5), and update interactively when a subset of genes 
993 is selected. The heatmap's rows and columns are ordered by co-clustering them to find latent

994 components, using the sklearn.cluster.bicluster.SpectralCoclustering implementation of the SVD-

995 based algorithm of (Dhillon, 2001).

996

\section{GO enrichment}

998 Below the essentiality score heatmap in the browser is the enrichment analysis panel, which 999 displays hypergeometric $p$-values for the selected gene set against various database annotation 1000 terms, as computed by gProfiler (Raudvere et al., 2019).

\section{Module heatmaps}

1003 To create heatmaps for each module (Table S3), the bias-corrected CERES scores for genes in 1004 the module were hierarchically biclustered with Ward linkage using the 1005 scipy.cluster.hierarchy.linkage function from the scipy Python package, with the method argument 1006 set to 'ward' and the optimal_ordering argument set to True. This biclustering was then visualized 1007 with the seaborn.clustermap function from the seaborn Python package.

1008

\section{Module GO term enrichments}

1010 In Table S3, GO term enrichment $p$-values were calculated via a hypergeometric test 1011 implemented using the scipy.stats.hypergeom.sf function from the scipy Python package. When 1012 calculating enrichments and $p$-values, genes not found in any module were excluded. GO term 1013 enrichments and $p$-values were calculated for all GO terms from the GO consortium (Ashburner 1014 et al., 2000; The Gene Ontology Consortium, 2017), except for GO terms with fewer than 20 total 1015 genes across all modules and three overly broad GO terms (biological process:biological process, 1016 cellular component:cellular component and molecular function:molecular function), which were 1017 excluded. The top 3 most-enriched GO terms for each module were listed with their 
1018 hypergeometric $p$-values, provided the $p$-value was significant at a per-module Bonferroni

1019 threshold of 0.05 , corrected across all GO terms.

\section{Generation of HeLa cell lines expressing individual sgRNAs and tagged genes}

1022 HeLa cells were maintained on tissue culture plastic and cultured in DMEM supplemented with 1023100 units $/ \mathrm{mL}$ penicillin, $100 \mu \mathrm{g} / \mathrm{mL}$ streptomycin, and $10 \%$ fetal calf serum. Cells were passaged 1024 by incubation with Accutase, pelleting with centrifugation at $300 \mathrm{~g}$ for $5 \mathrm{~min}$ at room temperature, 1025 and replating in fresh growth medium. To transduce cells with individual constructs, HeLa cells 1026 stably expressing Cas9-BFP were lentivirally infected with constructs expressing either individual 1027 sgRNAs or GFP- or mCherry-tagged genes and a puromycin resistance (PuroR) or blasticidin 1028 resistance (BlastR) gene. At $3 \mathrm{~d}$ after infection, cells were selected with $2 \mu \mathrm{g} / \mathrm{mL}$ puromycin or $102910 \mu \mathrm{g} / \mathrm{mL}$ blasticidin for $3 \mathrm{~d}$, and cultured for at least $3 \mathrm{~d}$ without selection agent before use in 1030 experiments.

\section{Immunoblotting}

1033 Cleared cell extracts prepared in lysis buffer (50 mM Tris-Hcl pH 7.5, 150 mM NaCl, 1mM EDTA, $10341 \%$ Triton X-100, 1 x cOmplete protease inhibitor cocktail (Roche) were heated in SDS loading 1035 buffer and subjected to SDS-PAGE, transferred to nitrocellulose, blotted and imaged using an 1036 Odyssey CLx (LI-COR Biosciences) or Supersignal West Femto Maximum Sensitivity Substrate 1037 with a Chemidoc System (Bio-Rad). The following antibodies were used: Rabbit polyclonal anti1038 TMEM189 (HPA059549, Sigma, 1:250), mouse monoclonal anti-GAPDH (AM4300, Fisher), 1039 rabbit polyclonal anti-mCherry (ab167453, Abcam), mouse monoclonal anti-GFP (A-11120, 1040 Thermo Fisher) and rabbit polyclonal anti-beta actin (ab8227, Abcam). In Figure 4F, the species 1041 shown is the predominant species detected using this antibody in RAW264.7 cells and HeLa cells, 1042 and corresponds to the predicted molecular weight of the TMEM189-UBE2V1 fusion. Cell extracts 1043 from HeLa-Cas9 cells expressing sgRNAs targeting either control loci or the TMEM189 locus 
1044 (with $>70 \%$ knockout efficiency verified using ICE analysis (Synthego)) were used to validate the 1045 specificity of the anti-TMEM189 antibody (data not shown).

\section{Time-lapse microscopy for transferrin endocytosis}

1048 HeLa cells that had been transduced with sgRNAs, co-expressed with GFP and PuroR, and 1049 selected with puromycin as described above were lifted, centrifuged, and re-plated in 24-well 1050 tissue culture plates in quadruplicate at a density of 50,000 cells per well. After $1 \mathrm{~d}$, cells were 1051 washed once in dPBS, incubated in dPBS for 30 minutes, and incubated in dPBS containing $105225 \mu \mathrm{g} / \mathrm{mL}$ transferrin-pHrodo (Thermo Fisher). Plates were transferred to an incubator and imaged

1053 every 20 minutes using an Incucyte (Essen). Total red intensity for each well, averaged over 16 1054 images per well, was calculated after applying a threshold of 1 RCU using top-hat background 1055 subtraction. Reported values represent the mean total red fluorescence intensity, normalized to 1056 the total green fluorescence signal to account for small variations in plating density, of triplicate 1057 wells. Similar results were obtained in three independent experiments using two sets of 1058 independently-generated cell lines.

\section{Confocal microscopy}

1061 HeLa cells were transduced with a lentiviral construct coexpressing C15orf57-GFP and PuroR, 1062 and then transduced with a lentiviral construct expressing AP2S1-mCherry and BlastR. Cells 1063 cultured in glass-bottom 24-well plates and imaged in a single plane near the glass surface using 1064 an inverted Nikon Eclipse Ti-E spinning disk confocal microscope and an Andor Ixon3 EMCCD 1065 camera using an oil-immersion 100x objective (NA=1.45). Images were assembled and adjusted 1066 for brightness and contrast in Photoshop (Adobe). 


\section{Immunoprecipitation and mass spectrometry}

1071 For immunoprecipitations, HeLa cells that had been transduced with tagged constructs as 1072 described above were cultured in either T-150 flasks or $15 \mathrm{~cm}$ plates and harvested near 1073 confluency. Cell lysates for each cell line were prepared by detaching cells with trypsin, washing 1074 in PBS, resuspending in $1 \mathrm{~mL}$ IP buffer (50 mM HEPES, pH 6.8, $150 \mathrm{mM} \mathrm{NaCl}, 2 \mathrm{mM}$ EDTA, 1\% 1075 Triton X-100, 1x cOmplete protease inhibitor cocktail (Roche)) and incubating for 30 min on ice. 1076 Cell lysates were cleared by centrifugation at 5,000 g for $5 \mathrm{~min}$ before incubation with $50 \mu \mathrm{l}$ pre1077 washed GFP-TRAP MA beads (Chromotek) for $1 \mathrm{~h}$ at 4 degrees Centigrade, with end-over-end 1078 rotation. Beads were washed 4 times for 5 min with $1 \mathrm{~mL}$ IP buffer prior to elution with $30 \mu$ SDS 1079 sample buffer at 70 degrees Centigrade. In Figure 5E, a similar procedure was followed, except 1080 RFP-TRAP MA beads (Chromotek) were used.

1082 For analysis by mass spectrometry, elutions were loaded on 4-12\% Bis-Tris NuPage SDS-PAGE 1083 gels (Thermo Fisher) and run at $100 \mathrm{~V}$ for 30 minutes. Gels were stained with SimplyBlue 1084 SafeStain (Thermo Fisher) and equivalent gel fragments for each lane were extracted, sliced into small fragments, and stored in $1 \%$ acetic acid. Samples were processed as described previously 1086 (Haney et al., 2018), with the following modifications. Briefly, gel slices were first resuspended in $1087100 \mu \mathrm{L} 50 \mathrm{mM}$ ammonium bicarbonate supplemented with $10 \mu \mathrm{l} 50 \mathrm{mM}$ dithiothreitol and 1088 incubated for $30 \mathrm{~min}$ at $55^{\circ} \mathrm{C}$, and subsequently alkylated with $10 \mu \mathrm{l} 100 \mathrm{mM}$ acrylamide for 30 1089 min at room temperature. Solution phase was discarded, and gel pieces were washed 3 times 1090 with 100 H 50 mM ammonium bicarbonate/50\% acetonitrile for $10 \mathrm{~min}$ at room temperature. 100 $1091 \mu \mathrm{L}$ of $50 \mathrm{mM}$ ammonium bicarbonate and $1 \mu \mathrm{g}$ trypsin was added to digest bound proteins during 1092 an overnight incubation at $37^{\circ} \mathrm{C}$. The overnight digests were spun down and the solution was 1093 collected. Peptides were extracted more two additional times with $50 \mu \mathrm{l}$ of $70 \%$ acetonitrile/29\% 1094 water $/ 1 \%$ formic acid and incubated for $10 \mathrm{~min}$ at $37^{\circ} \mathrm{C}$ and centrifuged at $10,000 \times g$ for 2 minutes, 1095 and all three extractions were combined. The combined extracts were dried using a Speedvac 
and reconstituted in $100 \mathrm{mM}$ triethylammonium bicarbonate for TMT10plex labelling (Thermo Fisher) following the manufacturer's instructions, and samples were mixed to generate the final

1098 peptide mixture.

1099

1100 Protein digests were loaded on a Waters Liquid Chromatography column coupled to an Orbitrap 1101 Fusion mass spectrometer (Thermo Fisher). Peptides were separated using a $25 \mathrm{~cm}$ long and $1102100 \mu \mathrm{m}$ inner diameter capillary column packed with Sepax $1.8 \mu \mathrm{m}$ C18 resin. Peptides were 1103 eluted off in a 60 min gradient at a flow rate of $600 \mathrm{nl} / \mathrm{min}$ from $5 \%$ to $35 \%$ acetonitrile in $0.1 \%$ 1104 formic acid. Mass spectrometry data was acquired by one full MS scan at $120 \mathrm{k}$ resolution followed 1105 with MS2 using HCD at 30k resolution. The instrument was set to run in top speed mode with $3 \mathrm{~s}$ 1106 cycle.

1108 Raw data was processed using Thermo Proteome Discoverer software version 2.2. MS data were 1109 searched against a human proteome database with $1 \%$ FDR at peptide level. Protein 1110 quantification was based on the precursor ion peak intensity using the label free quantitation 1111 workflow. For Figures 5D and S4B, keratins and proteins identified with only one peptide were 1112 excluded from analysis. P-values were generated from Student's t-tests between duplicate 1113 samples of indicated tagged genes and all 6 other samples analyzed in the same run (including 1114 duplicate samples derived from cells expressing GFP-tagged JTB (an unrelated gene), and from 1115 cells expressing GFP alone).

\section{$1117 \quad$ Lipidomics}

1118 HeLa cells expressing sgRNAs targeting either safe loci or the TMEM189 or SPTLC2 loci were 1119 cultured in quadruplicate and harvested by centrifugation after washing with PBS. Lipids were 1120 extracted from $60 \mathrm{mg}$ cell pellets using a biphasic separation with cold methyl tert-butyl ether 1121 (MTBE), methanol and water, as described previously (Schüssler-Fiorenza Rose et al., 2019). 
1122 The solvent mixture contained labeled standard lipids stock (SCIEX, cat\#: 5040156) to control for

1123 extraction efficiency and facilitate quantification relative to the known concentrations.

1125 Lipid extracts were analyzed by mass spectrometry using the Lipidyzer platform (Contrepois et 1126 al., 2018), comprising a 5500 QTRAP mass spectrometer equipped with a differential mobility 1127 scan (DMS) interface (SCIEX) and high-flow LC-30AD delivery unit (Shimadzu), as described 1128 previously (Schüssler-Fiorenza Rose et al., 2019). Briefly, flow injection analysis was performed 1129 at $8 \mu \mathrm{l} / \mathrm{min}$ in $10 \mathrm{mM}$ ammonium acetate in 50:50 dichloromethane:methanol running solution, with 1130 1-propanol included in curtain gas. DMS parameter settings were set as follows: 1131 Temperature $=$ Low, Separation Voltage $=3.5 \mathrm{kV}$ and DMS resolution $=$ Low. PC, PE, LPC, LPE 1132 were quantified with DMS on in negative ionization mode; SM was quantified with DMS on and in 1133 positive ionization mode; FFA were quantified with DMS off and in negative ionization mode; TAG, 1134 DAG, CE, and CER were quantified with DMS off and in positive ionization mode. DMS 1135 compensation voltages were tuned using a set of lipid standards (SCIEX, cat\#: 5040141), and a 1136 quick system suitability test (QSST) (SCIEX, cat\#: 50407) was performed to ensure acceptable 1137 limit of detection for each lipid class. Lipid molecular species were quantified with the Lipidyzer 1138 Workflow Manager using 54 deuterated IS developed with Avanti Polar Lipids covering 10 lipid 1139 classes (SCIEX, cat\#: 5040156). 17 plasmenylethanolamine species with fully saturated, 181140 carbon acyl chains at the sn-1 position were excluded from analyses, as they cannot be reliably 1141 differentiated from plasmanylethanolamine species with unsaturated 18-carbon acyl chains at the 1142 sn-1 position (which are scarce in wild-type cells but are expected to accumulate in TMEM1891143 knockout cells) with the Lipidyzer platform (M. Pearson, SCIEX, personal communication). 


\section{REFERENCES}

1148

1149

1150

1151

1152

1153

1154

1155

1156

1157

1158

1159

1160

1161

1162

1163

1164

1165

1166

1167

1168

Aitkin, A.C. (1935). On least squares and linear combination of observations. Proceedings of the Royal Society of Edinburgh 55, 42-48.

Alonso, J.M., and Ecker, J.R. (2006). Moving forward in reverse: genetic technologies to enable genome-wide phenomic screens in Arabidopsis. Nat. Rev. Genet. 7, 524-536.

Ascierto, P.A., Kirkwood, J.M., Grob, J.-J., Simeone, E., Grimaldi, A.M., Maio, M., Palmieri, G., Testori, A., Marincola, F.M., and Mozzillo, N. (2012). The role of BRAF V600 mutation in melanoma. J. Transl. Med. 10, 85.

Ashburner, M., Ball, C.A., Blake, J.A., Botstein, D., Butler, H., Cherry, J.M., Davis, A.P., Dolinski, K., Dwight, S.S., Eppig, J.T., et al. (2000). Gene ontology: tool for the unification of biology. The Gene Ontology Consortium. Nat. Genet. 25, 25-29.

Bakhoum, S.F., Ngo, B., Laughney, A.M., Cavallo, J.-A., Murphy, C.J., Ly, P., Shah, P., Sriram, R.K., Watkins, T.B.K., Taunk, N.K., et al. (2018). Chromosomal instability drives metastasis through a cytosolic DNA response. Nature 553, 467-472.

Barabási, A.-L., and Oltvai, Z.N. (2004). Network biology: understanding the cell's functional organization. Nat. Rev. Genet. 5, 101-113.

Bassik, M.C., Kampmann, M., Lebbink, R.J., Wang, S., Hein, M.Y., Poser, I., Weibezahn, J., Horlbeck, M.A., Chen, S., Mann, M., et al. (2013). A systematic mammalian genetic interaction map reveals pathways underlying ricin susceptibility. Cell 152, 909-922.

Blomen, V.A., Májek, P., Jae, L.T., Bigenzahn, J.W., Nieuwenhuis, J., Staring, J., Sacco, R., van Diemen, F.R., Olk, N., Stukalov, A., et al. (2015). Gene essentiality and synthetic lethality in haploid human cells. Science 350, 1092-1096. 
Boettcher, M., Tian, R., Blau, J.A., Markegard, E., Wagner, R.T., Wu, D., Mo, X., Biton, A., Zaitlen, N., Fu, H., et al. (2018). Dual gene activation and knockout screen reveals directional

1171 dependencies in genetic networks. Nat. Biotechnol. 36, 170-178.

1172 Boyle, E.A., Pritchard, J.K., and Greenleaf, W.J. (2018). High-resolution mapping of cancer cell 1173 networks using co-functional interactions. Mol. Syst. Biol. 14, e8594.

1174 Braverman, N., Steel, G., Obie, C., Moser, A., Moser, H., Gould, S.J., and Valle, D. (1997).

1175 Human PEX7 encodes the peroxisomal PTS2 receptor and is responsible for rhizomelic 1176 chondrodysplasia punctata. Nat. Genet. 15, 369-376.

1177 Breslow, D.K., and Weissman, J.S. (2010). Membranes in balance: mechanisms of sphingolipid 1178 homeostasis. Mol. Cell 40, 267-279.

1179 Breslow, D.K., Hoogendoorn, S., Kopp, A.R., Morgens, D.W., Vu, B.K., Kennedy, M.C., Han, K., 1180 Li, A., Hess, G.T., Bassik, M.C., et al. (2018). A CRISPR-based screen for Hedgehog signaling 1181 provides insights into ciliary function and ciliopathies. Nat. Genet. 50, 460-471.

1182 Carpenter, A.E., and Sabatini, D.M. (2004). Systematic genome-wide screens of gene function. 1183 Nat. Rev. Genet. 5, 11-22.

1184 Chan, E.M., Shibue, T., McFarland, J.M., Gaeta, B., Ghandi, M., Dumont, N., Gonzalez, A., 1185 McPartlan, J.S., Li, T., Zhang, Y., et al. (2019). WRN helicase is a synthetic lethal target in 1186 microsatellite unstable cancers. Nature 568, 551-556.

1187 Chen, J., and Wagner, E.J. (2010). snRNA 3' end formation: the dawn of the Integrator 1188 complex. Biochem. Soc. Trans. 38, 1082-1087.

1189 Choobdar, S., Ahsen, M.E., Crawford, J., Tomasoni, M., Fang, T., Lamparter, D., Lin, J., 1190 Hescott, B., Hu, X., Mercer, J., et al. (2019). Assessment of network module identification 
across complex diseases. Nat. Methods 16, 843-852.

1192 Chuang, H.-Y., Hofree, M., and Ideker, T. (2010). A Decade of Systems Biology. Annu. Rev. 1193 Cell Dev. Biol. 26, 721-744.

1194 Coifman, R.R., and Lafon, S. (2006). Diffusion maps. Applied and Computational Harmonic 1195 Analysis 21, 5-30.

1196 Collard, F., Baldin, F., Gerin, I., Bolsée, J., Noël, G., Graff, J., Veiga-da-Cunha, M., Stroobant, 1197 V., Vertommen, D., Houddane, A., et al. (2016). A conserved phosphatase destroys toxic 1198 glycolytic side products in mammals and yeast. Nat. Chem. Biol. 12, 601-607.

1199 Contrepois, K., Mahmoudi, S., Ubhi, B.K., Papsdorf, K., Hornburg, D., Brunet, A., and Snyder, M. (2018). Cross-Platform Comparison of Untargeted and Targeted Lipidomics Approaches on 1201 Aging Mouse Plasma. Sci. Rep. 8, 17747.

1202 Costanzo, M., Baryshnikova, A., Bellay, J., Kim, Y., Spear, E.D., Sevier, C.S., Ding, H., Koh, 1203 J.L.Y., Toufighi, K., Mostafavi, S., et al. (2010). The genetic landscape of a cell. Science 327, $1204 \quad 425-431$.

1205 Costanzo, M., VanderSluis, B., Koch, E.N., Baryshnikova, A., Pons, C., Tan, G., Wang, W., 1206 Usaj, M., Hanchard, J., Lee, S.D., et al. (2016). A global genetic interaction network maps a 1207 wiring diagram of cellular function. Science 353.

1208 Dhillon, I.S. (2001). Co-clustering Documents and Words Using Bipartite Spectral Graph 1209 Partitioning. In Proceedings of the Seventh ACM SIGKDD International Conference on 1210 Knowledge Discovery and Data Mining, (New York, NY, USA: ACM), pp. 269-274.

1211 Doench, J.G., Fusi, N., Sullender, M., Hegde, M., Vaimberg, E.W., Donovan, K.F., Smith, I., 1212 Tothova, Z., Wilen, C., Orchard, R., et al. (2016). Optimized sgRNA design to maximize activity 
and minimize off-target effects of CRISPR-Cas9. Nat. Biotechnol. 34, 184-191.

1214 Drew, K., Lee, C., Huizar, R.L., Tu, F., Borgeson, B., McWhite, C.D., Ma, Y., Wallingford, J.B., 1215 and Marcotte, E.M. (2017). Integration of over 9,000 mass spectrometry experiments builds a 1216 global map of human protein complexes. Mol. Syst. Biol. 13, 932.

1217 Du, D., Roguev, A., Gordon, D.E., Chen, M., Chen, S.-H., Shales, M., Shen, J.P., Ideker, T., 1218 Mali, P., Qi, L.S., et al. (2017). Genetic interaction mapping in mammalian cells using CRISPR 1219 interference. Nat. Methods 14, 577-580.

1220 Dutkowski, J., Kramer, M., Surma, M.A., Balakrishnan, R., Cherry, J.M., Krogan, N.J., and 1221 Ideker, T. (2013). A gene ontology inferred from molecular networks. Nat. Biotechnol. 31, 38122245.

1223 Fletcher, M.N.C., Castro, M.A.A., Wang, X., de Santiago, I., O’Reilly, M., Chin, S.-F., Rueda, 1224 O.M., Caldas, C., Ponder, B.A.J., Markowetz, F., et al. (2013). Master regulators of FGFR2 1225 signalling and breast cancer risk. Nat. Commun. 4, 2464.

1226 Furukawa, T., Tanji, E., Xu, S., and Horii, A. (2008). Feedback regulation of DUSP6 transcription responding to MAPK1 via ETS2 in human cells. Biochem. Biophys. Res. Commun. $1228377,317-320$.

1229 Gallego-García, A., Monera-Girona, A.J., Pajares-Martínez, E., Bastida-Martínez, E., Pérez-

1230 Castaño, R., Iniesta, A.A., Fontes, M., Padmanabhan, S., and Elías-Arnanz, M. (2019). A

1231 bacterial light response reveals an orphan desaturase for human plasmalogen synthesis.

1232 Science $366,128-132$.

1233 Gao, J., Ajjawi, I., Manoli, A., Sawin, A., Xu, C., Froehlich, J.E., Last, R.L., and Benning, C.

1234 (2009). FATTY ACID DESATURASE4 of Arabidopsis encodes a protein distinct from

1235 characterized fatty acid desaturases. Plant J. 60, 832-839. 
1236 Garraway, L.A., Widlund, H.R., Rubin, M.A., Getz, G., Berger, A.J., Ramaswamy, S.,

1237 Beroukhim, R., Milner, D.A., Granter, S.R., Du, J., et al. (2005). Integrative genomic analyses

1238 identify MITF as a lineage survival oncogene amplified in malignant melanoma. Nature 436,

$1239 \quad 117-122$.

1240 Guna, A., Volkmar, N., Christianson, J.C., and Hegde, R.S. (2018). The ER membrane protein

1241 complex is a transmembrane domain insertase. Science $359,470-473$.

1242

1243 Haghverdi, L., Buettner, F., and Theis, F.J. (2015). Diffusion maps for high-dimensional single-

1244 cell analysis of differentiation data. Bioinformatics 31, 2989-2998.

1245 Han, K., Jeng, E.E., Hess, G.T., Morgens, D.W., Li, A., and Bassik, M.C. (2017). Synergistic

1246 drug combinations for cancer identified in a CRISPR screen for pairwise genetic interactions.

1247 Nat. Biotechnol. 35, 463-474.

1248 Harris, M.L., Baxter, L.L., Loftus, S.K., and Pavan, W.J. (2010). Sox proteins in melanocyte

1249 development and melanoma. Pigment Cell Melanoma Res. 23, 496-513.

1250 Harrow, J., Frankish, A., Gonzalez, J.M., Tapanari, E., Diekhans, M., Kokocinski, F., Aken, B.L.,

1251 Barrell, D., Zadissa, A., Searle, S., et al. (2012). GENCODE: the reference human genome

1252 annotation for The ENCODE Project. Genome Res. 22, 1760-1774.

1253 Hartwell, L.H., Hopfield, J.J., Leibler, S., and Murray, A.W. (1999). From molecular to modular 1254 cell biology. Nature 402, C47-C52.

1255 Horlbeck, M.A., Xu, A., Wang, M., Bennett, N.K., Park, C.Y., Bogdanoff, D., Adamson, B.,

1256 Chow, E.D., Kampmann, M., Peterson, T.R., et al. (2018). Mapping the Genetic Landscape of

1257 Human Cells. Cell 174, 953-967.e22.

1258 Kang, Y., Baker, M.J., Liem, M., Louber, J., McKenzie, M., Atukorala, I., Ang, C.-S., 
Keerthikumar, S., Mathivanan, S., and Stojanovski, D. (2016). Tim29 is a novel subunit of the human TIM22 translocase and is involved in complex assembly and stability. Elife 5.

1261 Kim, E., Dede, M., Lenoir, W.F., Wang, G., Srinivasan, S., Colic, M., and Hart, T. (2019). A 1262 network of human functional gene interactions from knockout fitness screens in cancer cells.

1263 Life Sci Alliance 2.

1264 Kramer, M., Dutkowski, J., Yu, M., Bafna, V., and Ideker, T. (2014). Inferring gene ontologies 1265 from pairwise similarity data. Bioinformatics 30, i34-i42.

1266 Li, M.-T., Di, W., Xu, H., Yang, Y.-K., Chen, H.-W., Zhang, F.-X., Zhai, Z.-H., and Chen, D.-Y. 1267 (2013). Negative regulation of RIG-I-mediated innate antiviral signaling by SEC14L1. J. Virol. 1268 87, 10037-10046.

1269 Liu, Y., and Xie, J. (2019). Cauchy Combination Test: A Powerful Test With Analytic p-Value

1270 Calculation Under Arbitrary Dependency Structures. Journal of the American Statistical 1271 Association 1-18.

1272 Liu, Y., Chen, S., Li, Z., Morrison, A.C., Boerwinkle, E., and Lin, X. (2019). ACAT: A Fast and 1273 Powerful $p$ Value Combination Method for Rare-Variant Analysis in Sequencing Studies. The 1274 American Journal of Human Genetics 104, 410-421.

1275 McDonald, E.R., 3rd, de Weck, A., Schlabach, M.R., Billy, E., Mavrakis, K.J., Hoffman, G.R., 1276 Belur, D., Castelletti, D., Frias, E., Gampa, K., et al. (2017). Project DRIVE: A Compendium of 1277 Cancer Dependencies and Synthetic Lethal Relationships Uncovered by Large-Scale, Deep 1278 RNAi Screening. Cell 170, 577-592.e10.

1279 Mclnnes, L., Healy, J., Saul, N., and Großberger, L. (2018). UMAP: Uniform Manifold 1280 Approximation and Projection. Journal of Open Source Software 3, 861. 
1281 Meyers, R.M., Bryan, J.G., McFarland, J.M., Weir, B.A., Sizemore, A.E., Xu, H., Dharia, N.V.,

1282 Montgomery, P.G., Cowley, G.S., Pantel, S., et al. (2017). Computational correction of copy

1283 number effect improves specificity of CRISPR-Cas9 essentiality screens in cancer cells. Nat.

1284 Genet. 49, 1779-1784.

1285 Mohr, S.E., Smith, J.A., Shamu, C.E., Neumüller, R.A., and Perrimon, N. (2014). RNAi

1286 screening comes of age: improved techniques and complementary approaches. Nat. Rev. Mol.

1287 Cell Biol. 15, 591-600.

1288 Moll, U.M., and Petrenko, O. (2003). The MDM2-p53 Interaction. Mol. Cancer Res. 1, 100112891008.

1290 Motley, A., Bright, N.A., Seaman, M.N.J., and Robinson, M.S. (2003). Clathrin-mediated 1291 endocytosis in AP-2-depleted cells. J. Cell Biol. 162, 909-918.

1292 Nagan, N., and Zoeller, R.A. (2001). Plasmalogens: biosynthesis and functions. Prog. Lipid Res. $129340,199-229$.

1294 Nepusz, T., Yu, H., and Paccanaro, A. (2012). Detecting overlapping protein complexes in 1295 protein-protein interaction networks. Nat. Methods 9, 471-472.

1296 Okamura, Y., Aoki, Y., Obayashi, T., Tadaka, S., Ito, S., Narise, T., and Kinoshita, K. (2015).

1297 COXPRESdb in 2015: coexpression database for animal species by DNA-microarray and

1298 RNAseq-based expression data with multiple quality assessment systems. Nucleic Acids Res.

1299 43, D82-D86.

1300 Pan, J., Meyers, R.M., Michel, B.C., Mashtalir, N., Sizemore, A.E., Wells, J.N., Cassel, S.H., 1301 Vazquez, F., Weir, B.A., Hahn, W.C., et al. (2018). Interrogation of Mammalian Protein Complex 1302 Structure, Function, and Membership Using Genome-Scale Fitness Screens. Cell Syst 6, 5551303 568.e7. 
1304 Perotti, V., Baldassari, P., Molla, A., Vegetti, C., Bersani, I., Maurichi, A., Santinami, M.,

1305 Anichini, A., and Mortarini, R. (2016). NFATc2 is an intrinsic regulator of melanoma

1306 dedifferentiation. Oncogene 35, 2862-2872.

1307 Piano, V., Benjamin, D.I., Valente, S., Nenci, S., Marrocco, B., Mai, A., Aliverti, A., Nomura, 1308 D.K., and Mattevi, A. (2015). Discovery of Inhibitors for the Ether Lipid-Generating Enzyme 1309 AGPS as Anti-Cancer Agents. ACS Chem. Biol. 10, 2589-2597.

1310 Povey, S., Lovering, R., Bruford, E., Wright, M., Lush, M., and Wain, H. (2001). The HUGO

1311 Gene Nomenclature Committee (HGNC). Human Genetics 109, 678-680.

1312 Raudvere, U., Kolberg, L., Kuzmin, I., Arak, T., Adler, P., Peterson, H., and Vilo, J. (2019).

1313 g:Profiler: a web server for functional enrichment analysis and conversions of gene lists (2019 1314 update). Nucleic Acids Research 47, W191-W198.

1315 Rauscher, B., Heigwer, F., Henkel, L., Hielscher, T., Voloshanenko, O., and Boutros, M. (2018).

1316 Toward an integrated map of genetic interactions in cancer cells. Mol. Syst. Biol. 14, e7656.

1317 Ricke, R.M., van Ree, J.H., and van Deursen, J.M. (2008). Whole chromosome instability and 1318 cancer: a complex relationship. Trends Genet. 24, 457-466.

1319 Rickman, D.S., Schulte, J.H., and Eilers, M. (2018). The Expanding World of N-MYC-Driven 1320 Tumors. Cancer Discov. 8, 150-163.

1321 Roman, S.D., Ormandy, C.J., Manning, D.L., Blamey, R.W., Nicholson, R.I., Sutherland, R.L., 1322 and Clarke, C.L. (1993). Estradiol induction of retinoic acid receptors in human breast cancer 1323 cells. Cancer Res. 53, 5940-5945.

1324 Rosenbluh, J., Mercer, J., Shrestha, Y., Oliver, R., Tamayo, P., Doench, J.G., Tirosh, I., 1325 Piccioni, F., Hartenian, E., Horn, H., et al. (2016). Genetic and Proteomic Interrogation of Lower 

Syst 3, 302-316.e4.

Ruepp, A., Brauner, B., Dunger-Kaltenbach, I., Frishman, G., Montrone, C., Stransky, M., Waegele, B., Schmidt, T., Doudieu, O.N., Stümpflen, V., et al. (2008). CORUM: the comprehensive resource of mammalian protein complexes. Nucleic Acids Res. 36, D646-D650.

1333 Saxton, R.A., and Sabatini, D.M. (2017). mTOR Signaling in Growth, Metabolism, and Disease.

1334 Cell 169, 361-371.

1335 Schüssler-Fiorenza Rose, S.M., Contrepois, K., Moneghetti, K.J., Zhou, W., Mishra, T., 1336 Mataraso, S., Dagan-Rosenfeld, O., Ganz, A.B., Dunn, J., Hornburg, D., et al. (2019). A

1337 longitudinal big data approach for precision health. Nat. Med. 25, 792-804.

1338 Shalem, O., Sanjana, N.E., and Zhang, F. (2015). High-throughput functional genomics using 1339 CRISPR-Cas9. Nat. Rev. Genet. 16, 299-311.

1340 Shen, J.P., Zhao, D., Sasik, R., Luebeck, J., Birmingham, A., Bojorquez-Gomez, A., Licon, K., 1341 Klepper, K., Pekin, D., Beckett, A.N., et al. (2017). Combinatorial CRISPR-Cas9 screens for de 1342 novo mapping of genetic interactions. Nat. Methods $14,573-576$.

1343 Shoemaker, C.J., Huang, T.Q., Weir, N.R., Polyakov, N.J., Schultz, S.W., and Denic, V. (2019).

1344 CRISPR screening using an expanded toolkit of autophagy reporters identifies TMEM41B as a 1345 novel autophagy factor. PLoS Biol. 17, e2007044.

1346 Shurtleff, M.J., Itzhak, D.N., Hussmann, J.A., Schirle Oakdale, N.T., Costa, E.A., Jonikas, M., 1347 Weibezahn, J., Popova, K.D., Jan, C.H., Sinitcyn, P., et al. (2018). The ER membrane protein 
1348 complex interacts cotranslationally to enable biogenesis of multipass membrane proteins. Elife 13497.

1350 Snyder, F., Lee, T.-C., and Wykle, R.L. (1985). Ether-Linked Glycerolipids and Their Bioactive 1351 Species:Enzymes and Metabolic Regulation. In The Enzymes of Biological Membranes: Volume 1352 Biosynthesis and Metabolism, A.N. Martonosi, ed. (Boston, MA: Springer US), pp. 1-58.

1353 Storey, J.D., and Tibshirani, R. (2003). Statistical significance for genomewide studies. Proc. 1354 Natl. Acad. Sci. U. S. A. 100, 9440-9445.

1355 Stroud, D.A., Surgenor, E.E., Formosa, L.E., Reljic, B., Frazier, A.E., Dibley, M.G., Osellame, 1356 L.D., Stait, T., Beilharz, T.H., Thorburn, D.R., et al. (2016). Accessory subunits are integral for 1357 assembly and function of human mitochondrial complex I. Nature 538, 123-126.

1358 Stuart, J.M., Segal, E., Koller, D., and Kim, S.K. (2003). A gene-coexpression network for global 1359 discovery of conserved genetic modules. Science 302, 249-255.

1360 Szklarczyk, D., Morris, J.H., Cook, H., Kuhn, M., Wyder, S., Simonovic, M., Santos, A., 1361 Doncheva, N.T., Roth, A., Bork, P., et al. (2017). The STRING database in 2017: quality1362 controlled protein-protein association networks, made broadly accessible. Nucleic Acids Res. 1363 45, D362-D368.

1364 Szolderits, G., Daum, G., Paltauf, F., and Hermetter, A. (1991). Protein-catalyzed transport of 1365 ether phospholipids. Biochim. Biophys. Acta 1063, 197-202.

1366 Talbot, B.E., Vandorpe, D.H., Stotter, B.R., Alper, S.L., and Schlondorff, J.S. (2019).

1367 Transmembrane insertases and N-glycosylation critically determine synthesis, trafficking, and 1368 activity of the nonselective cation channel TRPC6. J. Biol. Chem. 294, 12655-12669.

1369 The Gene Ontology Consortium (2017). Expansion of the Gene Ontology knowledgebase and 
resources. Nucleic Acids Res. 45, D331-D338.

1371 Thomson, T.M., Lozano, J.J., Loukili, N., Carrió, R., Serras, F., Cormand, B., Valeri, M., Díaz,

1372 V.M., Abril, J., Burset, M., et al. (2000). Fusion of the human gene for the polyubiquitination

1373 coeffector UEV1 with Kua, a newly identified gene. Genome Res. 10, 1743-1756.

1374 Tian, S., Wu, Q., Zhou, B., Choi, M.Y., Ding, B., Yang, W., and Dong, M. (2019). Proteomic

1375 Analysis Identifies Membrane Proteins Dependent on the ER Membrane Protein Complex. Cell

1376 Reports 28, 2517-2526.e5.

1377 Tong, A.H.Y. (2004). Global Mapping of the Yeast Genetic Interaction Network. Science 303, 1378 808-813.

1379 Tong, A.H., Evangelista, M., Parsons, A.B., Xu, H., Bader, G.D., Pagé, N., Robinson, M., 1380 Raghibizadeh, S., Hogue, C.W., Bussey, H., et al. (2001). Systematic genetic analysis with 1381 ordered arrays of yeast deletion mutants. Science 294, 2364-2368.

1382 Tsherniak, A., Vazquez, F., Montgomery, P.G., Weir, B.A., Kryukov, G., Cowley, G.S., Gill, S., 1383 Harrington, W.F., Pantel, S., Krill-Burger, J.M., et al. (2017). Defining a Cancer Dependency 1384 Map. Cell 170, 564-576.e16.

1385 Volkmar, N., Thezenas, M.-L., Louie, S.M., Juszkiewicz, S., Nomura, D.K., Hegde, R.S., 1386 Kessler, B.M., and Christianson, J.C. (2019). The ER membrane protein complex promotes 1387 biogenesis of sterol-related enzymes maintaining cholesterol homeostasis. J. Cell Sci. 132.

1388 Wang, T., Yu, H., Hughes, N.W., Liu, B., Kendirli, A., Klein, K., Chen, W.W., Lander, E.S., and 1389 Sabatini, D.M. (2017). Gene Essentiality Profiling Reveals Gene Networks and Synthetic Lethal 1390 Interactions with Oncogenic Ras. Cell 168, 890-903.e15.

1391 Wang, X., Li, G., Koul, S., Ohki, R., Maurer, M., Borczuk, A., and Halmos, B. (2018). PHLDA2 is 
a key oncogene-induced negative feedback inhibitor of EGFR/ErbB2 signaling via interference with AKT signaling. Oncotarget 9, 24914.

1394 Xu, X., Xu, L., Gao, F., Wang, J., Ye, J., Zhou, M., Zhu, Y., and Tao, L. (2014). Identification of 1395 a novel gene fusion (BMX-ARHGAP) in gastric cardia adenocarcinoma. Diagn. Pathol. 9, 218.

1396 Yu, J., Pressoir, G., Briggs, W.H., Vroh Bi, I., Yamasaki, M., Doebley, J.F., McMullen, M.D., 1397 Gaut, B.S., Nielsen, D.M., Holland, J.B., et al. (2006). A unified mixed-model method for 1398 association mapping that accounts for multiple levels of relatedness. Nat. Genet. 38, $203-208$.

1399 Zhang, Y.-W., Nasto, R.E., Varghese, R., Jablonski, S.A., Serebriiskii, I.G., Surana, R., Calvert, 1400 V.S., Bebu, I., Murray, J., Jin, L., et al. (2016). Acquisition of estrogen independence induces 1401 TOB1-related mechanisms supporting breast cancer cell proliferation. Oncogene 35, 164314021656.

1403 Zhu, C., Wu, L., Lv, Y., Guan, J., Bai, X., Lin, J., Liu, T., Yang, X., Robson, S.C., Sang, X., et al. 1404 (2019). The fusion landscape of hepatocellular carcinoma. Mol. Oncol. 13, 1214-1225.

1405 Zoeller, R.A., Rangaswamy, S., Herscovitz, H., Rizzo, W.B., Hajra, A.K., Das, A.K., Moser, 1406 H.W., Moser, A., Lazarow, P.B., and Santos, M.J. (1992). Mutants in a macrophage-like cell line 1407 are defective in plasmalogen biosynthesis, but contain functional peroxisomes. J. Biol. Chem. $1408 \quad 267,8299-8306$. 


\section{SUPPLEMENTAL INFORMATION}

\section{Figure S1, related to Figure 1}

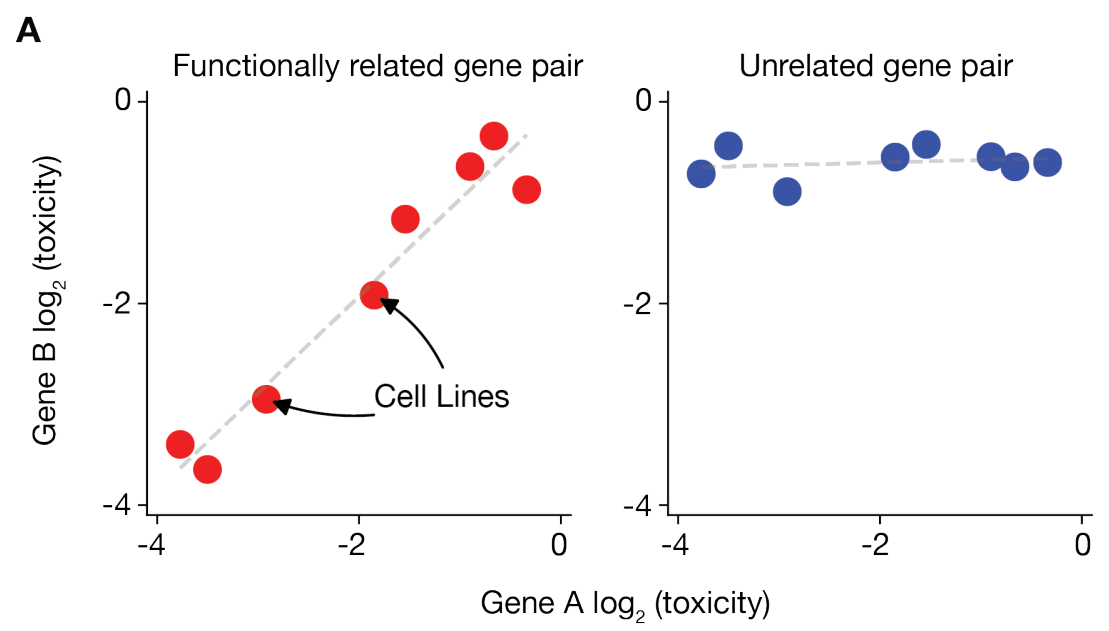

B

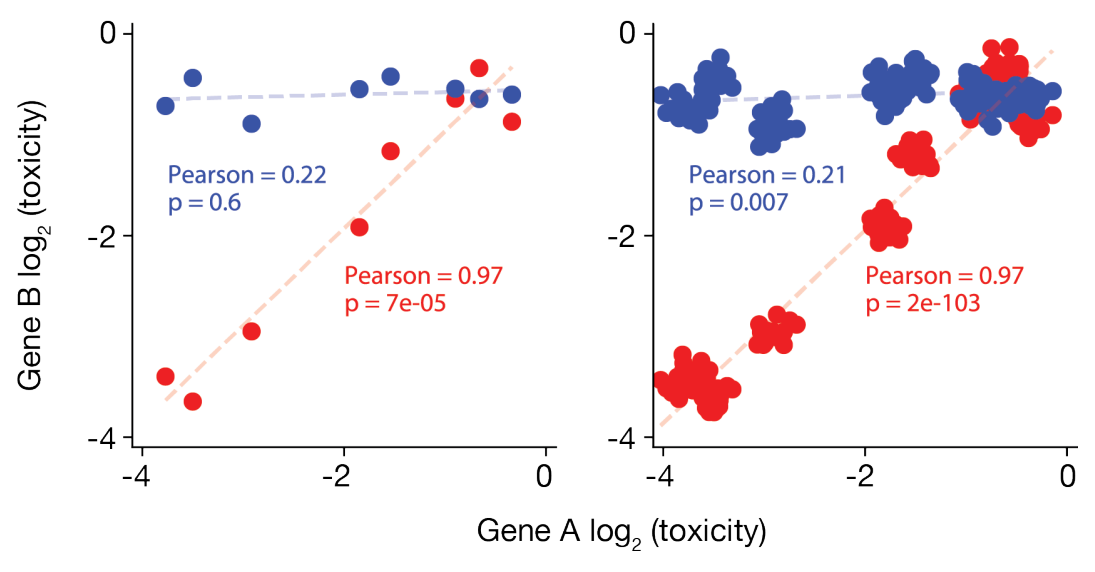


Figure S1: Co-essentiality profiling and the limitations of Pearson correlation

(A) The concept of co-essentiality: (left) a pair of functionally related genes are both essential in some cell lines and both non-essential in other lines. Essentiality can be quantified from CRISPR screens as the logarithm of the growth effect of the gene's knockout (intuitively, the number of times fewer cells with the knockout doubled during the screen, compared to control cells). (Right) a pair of unrelated genes have uncorrelated essentiality across cell lines.

(B) Simulation of how biological relatedness between cell lines inflates Pearson correlation $p$ values. Duplicating each point 10 times with slight noise (analogous to duplicating each screen in 10 related lines) makes the previously non-significant $(p=0.6)$ blue correlation highly significant $(p=0.007)$ and the significant red correlation $\left(p=7 \times 10^{-5}\right)$ substantially more so $\left(p=2 \times 10^{-103}\right)$, despite similar correlation magnitudes. 


\section{Figure S2, Related to Figure 2}
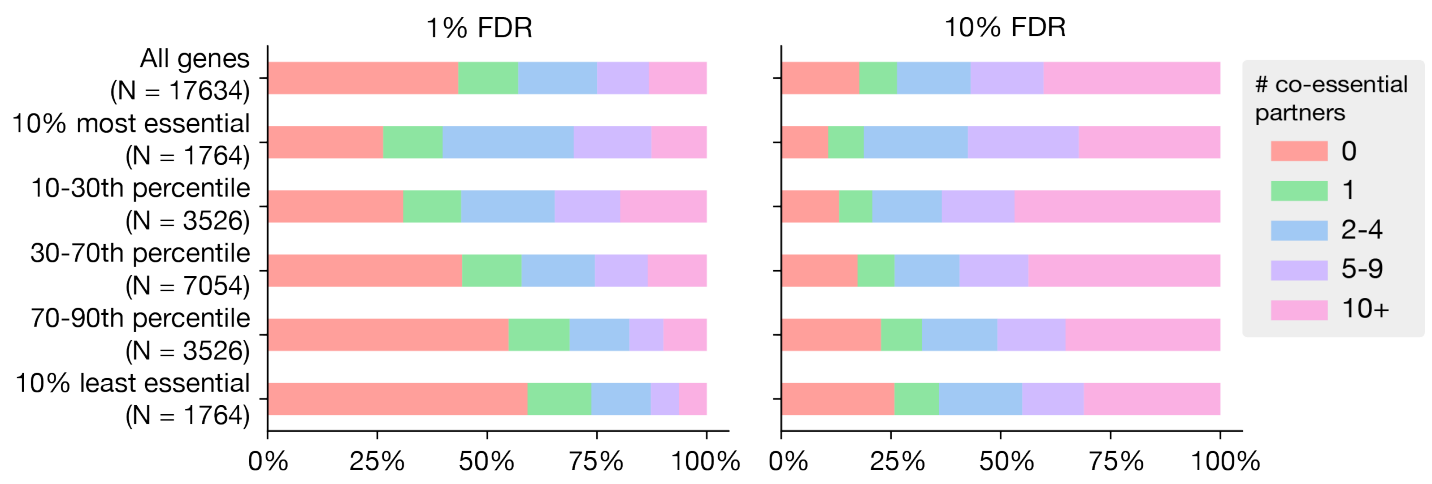
Figure S2: Number of co-essential partners per gene by average gene essentiality

Number of co-essential partners at $1 \%$ and $10 \%$ FDR as a function of a gene's average essentiality (pre-bias-correction CERES score) across lines. 


\section{Figure S3, Related to Figure 2}

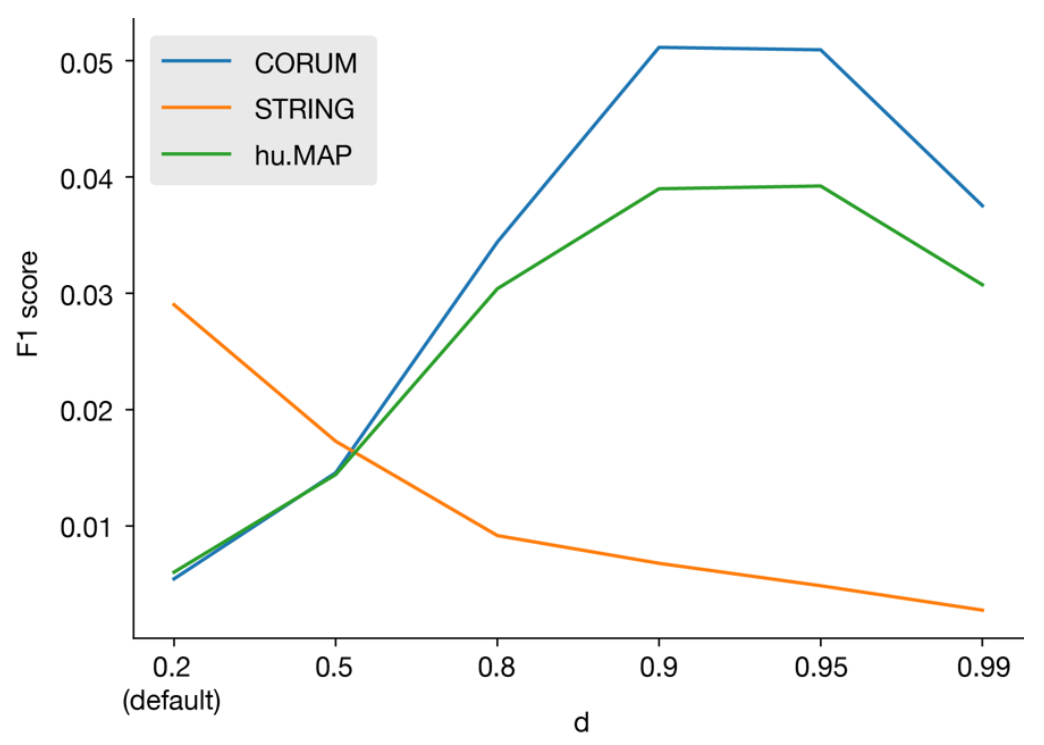




\section{Figure S3: Benchmarking of cluster density d}

F1 score (harmonic mean of precision and recall) for various values of the module density parameter $d$ on CORUM, hu.MAP and STRING. F1 scores represent the performance of a binary network based on the modules (i.e. "are genes A and B in the same module?") at predicting a binary network based on the benchmark dataset (i.e. "are genes A and B partners in the benchmark dataset?"). 


\section{Figure S4, Related to Figure 4}

A

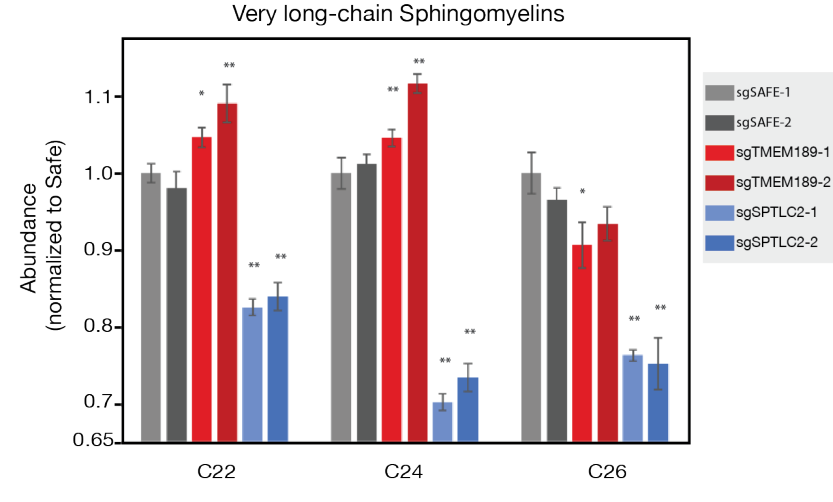

B

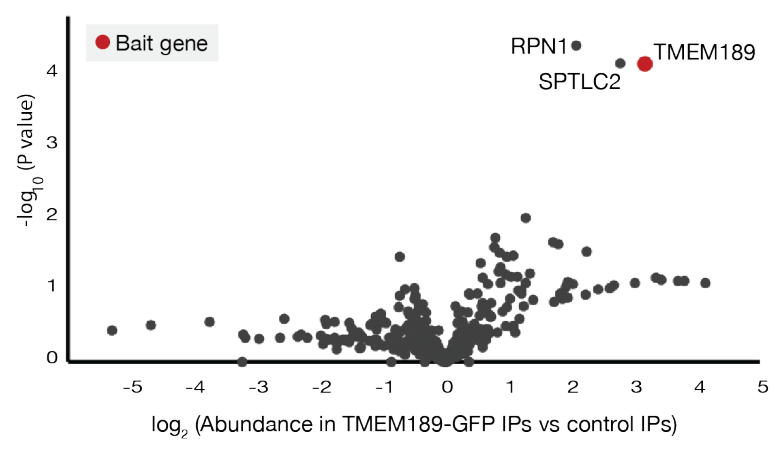


Figure S4: Additional functional characterization of TMEM189 suggests a secondary role in sphingolipid biosynthesis

(A) Abundances (relative to Safe-targeting sgRNA control \#1) of very long chain sphingomyelin species (with acyl chain length indicated on x-axis) in cell extracts prepared from HeLa cells transduced with indicated sgRNAs. sgSafe data and sgTMEM189 data are from same data set represented in Figure 4C. Error bars represent standard deviation ( $n=4$ technical replicates, twotailed Student's t-test, $\left.{ }^{*}, \mathrm{p}<.05 ;{ }^{* *} \mathrm{p}<.01\right)$.

(B) Volcano plot of mass spectrometric (TMT) analysis of TMEM189-GFP immunoprecipitates. Data are from same mass spectrometry analysis as data shown in Figure 4D. 
Figure S5, Related to Figure 6

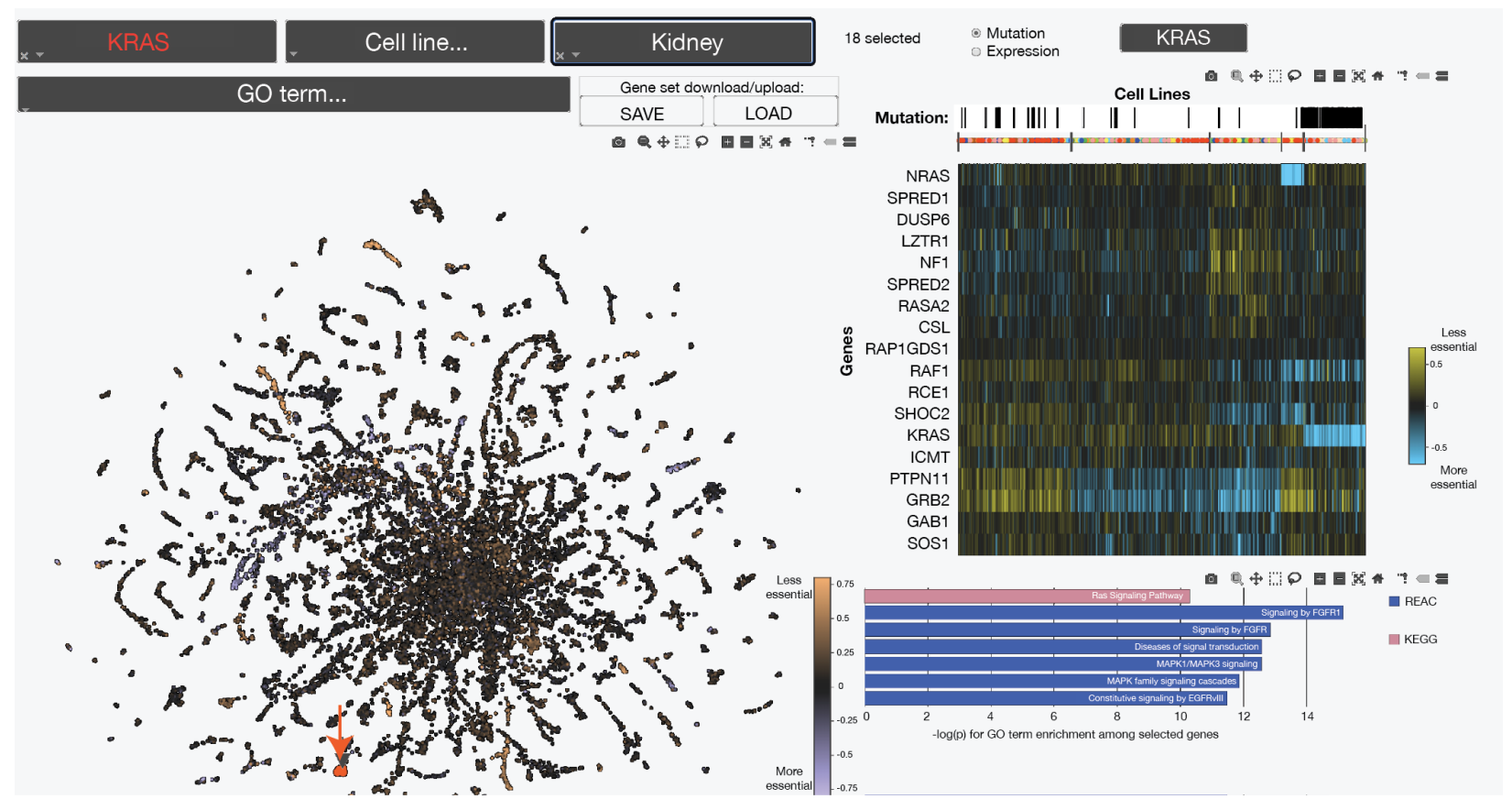




\section{Figure S5: A web tool for interactive exploration of the co-essential network}

Example use case for the interactive web tool (coessentiality.net). A gene, KRAS, was selected using the dropdown menu at top left and is marked with a red arrow in the scatterplot below. Genes selected for analysis - KRAS and its gene neighborhood - are designated with red points in the main panel (left). The heatmap panel (top right) shows that KRAS-mutant lines (selected for display using the search bar above the heat map and indicated as black marks in the "Mutation" bar above the heatmap) are enriched in a cluster (far right) that is marked by increased essentiality of KRAS. The pathway enrichment panel (bottom right) shows strong enrichments for Ras signaling and related pathways. The points in the main panel have also been selected in the tissue search bar (top middle) to be colored according to the average essentialities of each gene in kidney-derived cell lines. Gene sets can also be either saved or uploaded as csv files using the respective buttons in the top center (under "Gene set download/upload"). Some web colors and font sizes were optimized for display in this figure. 


\section{Figure S6, Related to Figure 2}
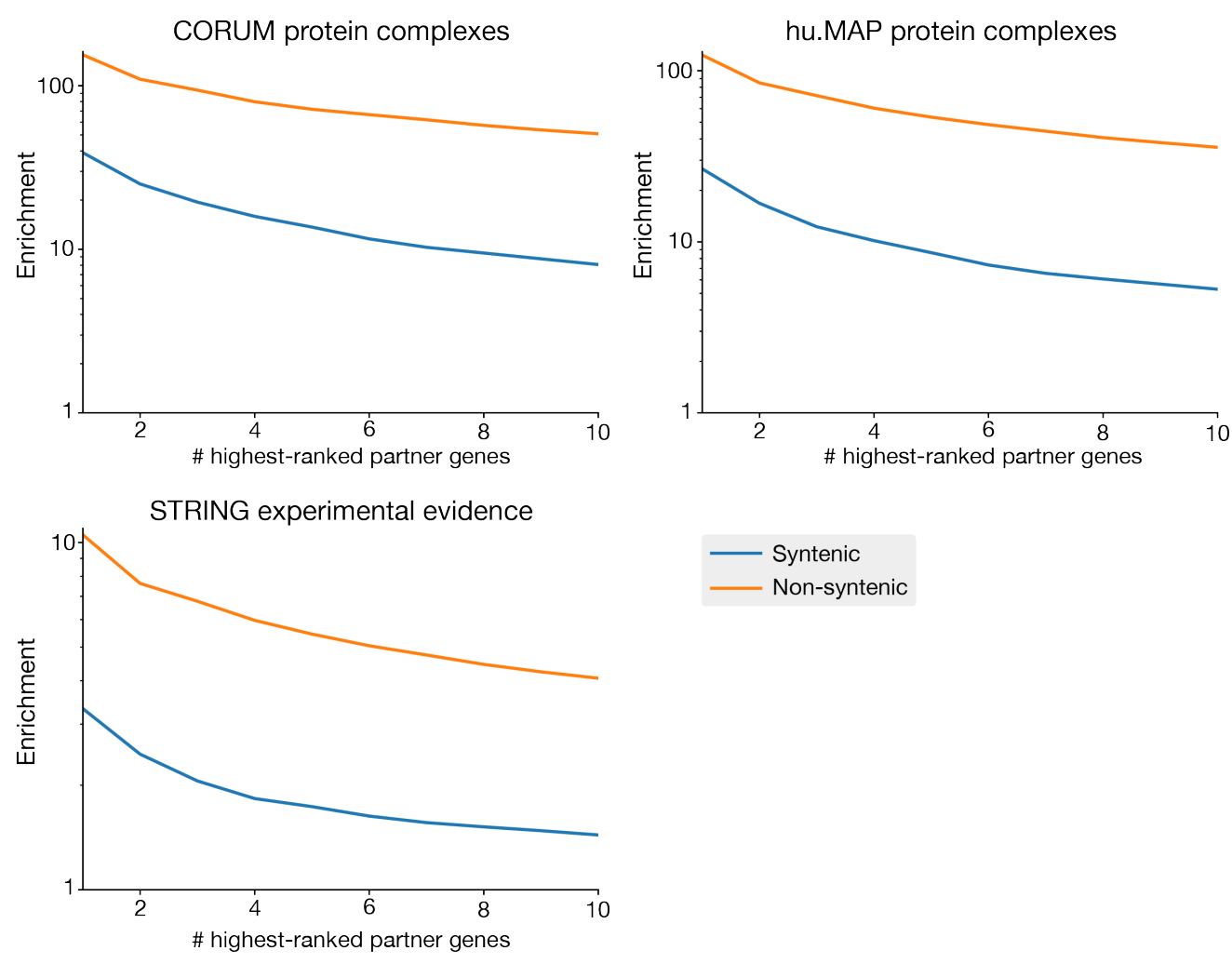
Figure S6: Benchmarking of syntenic versus non-syntenic modules

Enrichment of syntenic (both genes on same chromosome) and non-syntenic co-essential pairs

for annotated interactions CORUM, hu.MAP and STRING databases, using the same benchmarking strategy as in Figure 2. 

not certified by peer review) is the author/funder. All rights reserved. No reuse allowed without permission.

Figure S7, Related to Figure 2

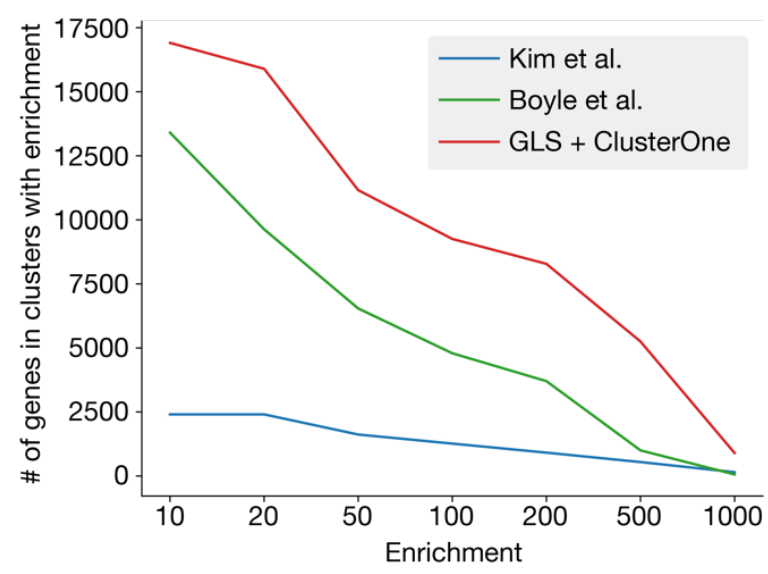


Figure S7: Number of genes assigned putative functions by various co-essentiality module detection methods, after excluding syntenic modules.

Number of genes in non-syntenic clusters/modules at least $\mathrm{N}$-fold enriched for some GO term, excluding the gene itself from the enrichment calculation, for various $\mathrm{N}$ from 10 to 1000 . 
Table S1: Spreadsheet of significant co-essential interactions at 10\% per-gene FDR.

List of all co-essential gene pairs identified in this study, with the number of Pubmed citations (as of Oct 2019) and chromosome location for each gene, and the direction of the gene correlation (positive (+) or negative (-)).

Table S2: Co-essential and co-expressed partners of TP53, KRAS and BRCA1.

\begin{tabular}{|c|c|c|c|c|c|}
\hline \multirow{2}{*}{$\frac{\text { Gene }}{\text { TP53 }}$} & \multicolumn{3}{|c|}{$\begin{array}{l}\text { Significant co-essential } \\
\text { partners ( } p \text {-value, direction) }\end{array}$} & \multicolumn{2}{|c|}{$\begin{array}{l}\text { Top co-expressed partners } \\
\text { (correlation) }\end{array}$} \\
\hline & USP28 & $3 \times 10^{-28}$ & + & CPEB4 & -0.32 \\
\hline & CDKN1A & $1 \times 10^{-24}$ & + & SYNJ1 & -0.32 \\
\hline & TP53BP1 & $5 \times 10^{-23}$ & + & PFN1 & 0.32 \\
\hline & MDM2 & $4 \times 10^{-18}$ & - & RNPEP & 0.31 \\
\hline & CHEK2 & $2 \times 10^{-16}$ & + & RCC2 & 0.31 \\
\hline & ATM & $1 \times 10^{-14}$ & + & CNN2 & 0.30 \\
\hline & PPM1D & $2 \times 10^{-9}$ & - & SERINC1 & -0.30 \\
\hline & XPO7 & $6 \times 10^{-8}$ & + & FAM126B & -0.30 \\
\hline & UBE2K & $9 \times 10^{-6}$ & + & FOXM1 & 0.30 \\
\hline & CNOT2 & $1 \times 10^{-5}$ & - & CHST14 & 0.30 \\
\hline \multirow[t]{5}{*}{ KRAS } & RAF1 & $1 \times 10^{-12}$ & + & ZDHHC2O & 0.55 \\
\hline & DOCK5 & $4 \times 10^{-7}$ & + & PTAR1 & 0.49 \\
\hline & SHOC2 & $2 \times 10^{-6}$ & + & MATR3 & 0.49 \\
\hline & ERGIC2 & $4 \times 10^{-6}$ & + & SPCS3 & 0.47 \\
\hline & TM7SF3 & $8 \times 10^{-6}$ & + & SUZ12 & 0.47 \\
\hline \multirow[t]{6}{*}{ BRCA1 } & BARD1 & $1 \times 10^{-25}$ & + & KIF14 & 0.67 \\
\hline & PALB2 & $2 \times 10^{-8}$ & + & MCM10 & 0.65 \\
\hline & RPL21 & $1 \times 10^{-6}$ & - & KIF11 & 0.63 \\
\hline & BRCA2 & $2 \times 10^{-5}$ & + & FANCD2 & 0.63 \\
\hline & HIST2H2AA3 & $4 \times 10^{-5}$ & - & NCAPH & 0.62 \\
\hline & HEY1 & $6 \times 10^{-5}$ & + & ARHGAP11A & 0.62 \\
\hline
\end{tabular}

Significant GLS co-essential versus top co-expressed partners of TP53, KRAS and BRCA1. Genes in bold have strong evidence of being part of the same pathway. 
Table S3: Spreadsheet of co-essential modules.

List of all 5,228 co-essential modules and their constituent genes, with top 3 most-enriched gene ontology terms and their associated enrichments and $p$-values, the value of $d$ used to define the module, and a link to the heatmap of batch-corrected essentiality data across 485 cell lines.

\section{Table S4: Uncharacterized gene functional predictions.}

List of uncharacterized genes that are present in co-essential modules $>100$-fold enriched for a gene ontology term, the Uniprot annotation score and number of Pubmed citations for each gene (as of Oct 2019), and the set of genes in each cluster that is and is not annotated with the mostenriched gene ontology term.

\section{Table S5: Lipidomics data.}

Lipid species concentrations for indicated lipids measured using Lipidyzer platform in indicated cell lines. QC1, QC2, and QC3 indicate quality controls (see Methods).

\section{Table S6: Mass spectrometry data for proteomic analysis of C15orf57 and TMEM189 interactomes.}

Proteomic data, including complete list of proteins and enrichment $p$-values, for $\mathrm{C} 15$ orf57 and TMEM189 interactome analyses in Figures 4 and 5.

\section{Table S7: Cancer type-specific module dependencies.}

List of 444 differentially essential modules across 16 tissue types, ranked by $p$-value. 
Video S1: Example use cases of co-essential browser.

Guide to use of co-essential browser showing how to navigate web tool in the context of multiple use cases, including gene lookup, gene set selection, and gene list upload. 